\title{
Neuropeptide Y Expression Defines a Novel Class of GABAergic Projection Neuron in the Inferior Colliculus
}

\author{
${ }^{\circledR}$ Marina A. Silveira, ${ }^{1}$ Justin D. Anair, ${ }^{1}{ }^{\circledR}$ Nichole L. Beebe, ${ }^{2}$ Pooyan Mirjalili, ${ }^{2}{ }^{\circledR}$ Brett R. Schofield, ${ }^{2}$ and \\ ${ }^{-}$Michael T. Roberts ${ }^{1}$ \\ ${ }^{1}$ Kresge Hearing Research Institute, Department of Otolaryngology-Head and Neck Surgery, University of Michigan, Ann Arbor, Michigan 48109, \\ and ${ }^{2}$ Department of Anatomy and Neurobiology, Northeast Ohio Medical University, Rootstown, Ohio 44272
}

Located in the midbrain, the inferior colliculus (IC) integrates information from numerous auditory nuclei and is an important hub for sound processing. Despite its importance, little is known about the molecular identity and functional roles of defined neuron types in the IC. Using a multifaceted approach in mice of both sexes, we found that neuropeptide Y (NPY) expression identifies a major class of inhibitory neurons, accounting for approximately one-third of GABAergic neurons in the IC. Retrograde tracing showed that NPY neurons are principal neurons that can project to the medial geniculate nucleus. In brain slice recordings, many NPY neurons fired spontaneously, suggesting that NPY neurons may drive tonic inhibition onto postsynaptic targets. Morphologic reconstructions showed that NPY neurons are stellate cells, and the dendrites of NPY neurons in the tonotopically organized central nucleus of the IC cross isofrequency laminae. Immunostaining confirmed that NPY neurons express NPY, and we therefore hypothesized that NPY signaling regulates activity in the IC. In crosses between $N p y 1 r^{c r e}$ and Ail4 Cre-reporter mice, we found that NPY $\mathrm{Y}_{1}$ receptor $\left(\mathrm{Y}_{1} \mathrm{R}\right)$-expressing neurons are glutamatergic and were broadly distributed throughout the rostrocaudal extent of the IC. In whole-cell recordings, application of a high-affinity $\mathrm{Y}_{1} \mathrm{R}$ agonist led to hyperpolarization in most $\mathrm{Y}_{1} \mathrm{R}$-expressing IC neurons. Thus, NPY neurons represent a novel class of inhibitory principal neurons that are well poised to use GABAergic and NPY signaling to regulate the excitability of circuits in the IC and auditory thalamus.

Key words: auditory; GABAergic; inferior colliculus; neuropeptide Y; projection neuron; Y1 receptor

Significance Statement

The identification of neuron types is a fundamental question in neuroscience. In the inferior colliculus (IC), the hub of the central auditory pathway, molecular markers for distinct classes of inhibitory neurons have remained unknown. We found that neuropeptide Y (NPY) expression identifies a class of GABAergic principal neurons that constitute one-third of the inhibitory neurons in the IC. NPY neurons fire spontaneously, have a stellate morphology, and project to the auditory thalamus. Additionally, we found that NPY signaling hyperpolarized the membrane potential of a subset of excitatory IC neurons that express the NPY $Y_{1}$ receptor. Thus, NPY neurons are a novel class of inhibitory neurons that use GABA and NPY signaling to regulate activity in the IC and auditory thalamus.

\section{Introduction}

The inferior colliculus (IC) is the hub of the central auditory pathway (Adams, 1979; Cant and Benson, 2006, 2007), a critical

\footnotetext{
Received Feb. 23, 2020; revised Apr. 22, 2020; accepted May 1, 2020.

Author contributions: M.A.S., N.L.B., B.R.S., and M.T.R. designed research; M.A.S., J.D.A., N.L.B., and P.M. performed research; M.A.S., J.D.A., N.L.B., P.M., B.R.S., and M.T.R. analyzed data; M.A.S., N.L.B., B.R.S., and M.T.R. wrote the first draft of the paper; M.A.S., J.D.A., N.L.B., B.R.S., and M.T.R. edited the paper; M.A.S., N.L.B., B.R.S., and M.T.R. wrote the paper.

The authors declare no competing financial interests.

This work was supported by American Hearing Research Foundation Research Grant to M.A.S.; and National Institutes of Health Grant R56 DC016880 to M.T.R. and Grant R01 DC004391 to B.R.S. We thank Bo Duan for helpful discussions and advice.

Correspondence should be addressed to Michael T. Roberts at microb@umich.edu.

https://doi.org/10.1523/JNEUROSCI.0420-20.2020

Copyright $\odot 2020$ the authors
}

processing center for most aspects of hearing, and an important site of plasticity after hearing loss (Chambers et al., 2016; Sturm et al., 2017). Despite its importance in auditory processing, it has proven difficult to identify the neuron classes that comprise the IC (Peruzzi et al., 2000; Palmer et al., 2013; Beebe et al., 2016). As a result, the cellular organization and function of neural circuits in the IC remain largely unclear.

The IC contains three main subdivisions: the central nucleus (ICc), dorsal cortex (ICd), and lateral cortex (IClc) (Morest and Oliver, 1984; Faye-Lund and Osen, 1985). The ICc is tonotopically organized into isofrequency laminae, and neurons in the ICc are divided in two broad morphologic families: disk-shaped and stellate neurons (Meininger et al., 1986; Malmierca et al., 1993). Disk-shaped neurons, the majority of neurons, maintain their dendritic arbors within isofrequency laminae (Oliver and 
Morest, 1984), whereas stellate neurons extend their dendritic arbors across isofrequency laminae and integrate information across sound frequencies (Oliver et al., 1991). However, there is considerable diversity within the disk-shaped and stellate groups, suggesting that they contain multiple neuron types (Oliver et al., 1994; Peruzzi et al., 2000; Ono et al., 2005; Wallace et al., 2012).

GABAergic neurons represent $\sim 25 \%$ of neurons in the IC (Oliver et al., 1994; Merchán et al., 2005; Beebe et al., 2016). In addition to providing local inhibition, many GABAergic neurons project to the medial geniculate nucleus (MG, auditory thalamus) (Winer et al., 1996; Peruzzi et al., 1997). Anatomical studies have subdivided GABAergic neurons into classes based on soma size and extracellular markers (Ito et al., 2009; Beebe et al., 2016), and neurons in all these classes can project to the MG (Beebe et al., 2018). However, it is unclear whether these anatomically defined groups represent neuron classes with distinct functional roles.

In many brain regions, an approach that combines anatomic and physiological analyses with molecular markers has proven key to identifying functionally distinct neuron types (Tremblay et al., 2016; Zeng and Sanes, 2017). Using such an approach, we recently identified vasoactive intestinal peptide (VIP) as a marker for a distinct class of excitatory principal neurons in the IC (Goyer et al., 2019), but there are currently no known molecular markers for GABAergic neuron classes in the IC.

Neuropeptide Y (NPY) is a 36 amino-acid neuropeptide and one of the most abundant peptides in the brain (Chronwall et al., 1985; O'Donohue et al., 1985). NPY is released from neurons and binds to the NPY family of $\mathrm{G}_{\mathrm{i} / \mathrm{o}}$-coupled G-protein coupled receptors (Dumont et al., 1998a). NPY signaling modulates synaptic transmission, neuronal excitability, and other physiological processes (Bacci et al., 2002). Across several brain regions, NPY has proven to be an important molecular marker for distinct classes of GABAergic neurons (Tong et al., 2008; Polgár et al., 2011). Because previous studies suggest that NPY (Nakagawa et al., 1995) and NPY $Y_{1}$ receptors $\left(\mathrm{Y}_{1} \mathrm{R}\right)$ are expressed in the IC (Kishi et al., 2005; Eva et al., 2006), we hypothesized that NPY is a molecular marker for a class of GABAergic neurons in the IC.

Using a multifaceted approach, we found that NPY is expressed by a distinct population of GABAergic neurons in the IC. These neurons have a stellate morphology and represent approximately one-third of the GABAergic neurons in the IC. Retrograde tracing showed that NPY neurons can project to the MG. In brain slice recordings, NPY neurons fired spontaneously, suggesting a tonic release of NPY and GABA. Using Npy1r $r^{\text {cre }}$ mice, we found that $\mathrm{Y}_{1} \mathrm{R}$-expressing IC neurons are glutamatergic and that application of a $\mathrm{Y}_{1} \mathrm{R}$-selective agonist hyperpolarized the membrane potential of most $\mathrm{Y}_{1} \mathrm{R}$-expressing neurons. Thus, our data indicate that NPY neurons represent a novel class of GABAergic IC principal neurons that may use GABAergic and NPY signaling to regulate the excitability of postsynaptic targets.

\section{Materials and Methods}

\section{Animals}

All experiments were approved by the University of Michigan Institutional Animal Care and Use Committee and were in accordance with National Institutes of Health's Guide for the care and use of laboratory animals. Animals were kept on a $12 \mathrm{~h}$ day/night cycle with ad libitum access to food and water. NPY-hrGFP mice were obtained from The Jackson Laboratory (stock \#006417) (van den Pol et al., 2009) and were maintained hemizygous for the hrGFP transgene by crossing with C57BL/ 6J mice (The Jackson Laboratory, stock \#000664). To visualize neurons that express $\mathrm{Y}_{1} \mathrm{R}, N p y 1 r^{\text {cre }}$ mice, B6.Cg-Npy1r ${ }^{\text {tm1.1(cre/GFP)Rpa }}$ (The Jackson
Laboratory, stock \#030544) (Padilla et al., 2016) were crossed with Ai14 Cre-reporter mice (B6.Cg-Gt(ROSA)26Sor ${ }^{\text {tm14(CAG-tdTomato)Hze; The }}$ Jackson Laboratory, stock \#007914). All mouse lines were on a C57BL/6J background. Because C57BL/6J mice are subject to age-related hearing loss due to the Cdh23 ${ }^{\text {ahl }}$ mutation (Noben-Trauth et al., 2003), experiments were performed in animals aged P22-P74, before the onset of hearing loss symptoms at $\sim 3$ months of age. For all experiments, mice of either sex were used.

\section{Analysis of the distribution of NPY neurons}

Three NPY-hrGFP mice were used to assess the distribution of NPY neurons in the IC. Each hrGFP-expressing IC neuron was identified in a series of every third section through the rostrocaudal extent of one IC. The locations of the labeled neurons were plotted using a Neurolucida system (MBF Bioscience) attached to an Axio Imager.Z2 microscope (Carl Zeiss). IC subdivisions were determined by examining staining for GAD67 and GlyT2 (Buentello et al., 2015). Low-magnification photomicrographs of the distribution of NPY neurons were collected using a $5 \times$ objective on an Axio Imager.Z2 microscope (Carl Zeiss). Neurolucida Explorer was used to export data to Excel for analysis.

\section{Immunohistochemistry}

Mice were deeply anesthetized and perfused transcardially with PBS (0.01 $\mathrm{M} \mathrm{PB}$ in $0.9 \% \mathrm{NaCl}), \mathrm{pH} 7.4$, for $1 \mathrm{~min}$ and then with a $10 \%$ buffered formalin solution (Sigma Millipore, catalog \#HT501128) for $15 \mathrm{~min}$. Brains were collected and postfixed in the same fixative for $2 \mathrm{~h}$ and cryoprotected overnight at $4^{\circ} \mathrm{C}$ in PBS containing $20 \%$ sucrose. Brains were cut into $40 \mu \mathrm{m}$ sections on a vibratome or freezing microtome. Sections were washed in PBS, and then treated with $10 \%$ normal donkey serum (Jackson ImmunoResearch Laboratories, catalog \#017$000-121$ ) and $0.3 \%$ Triton X-100 for $2 \mathrm{~h}$. Sections were then incubated for $24 \mathrm{~h}$ at $4^{\circ} \mathrm{C}$ in mouse anti-GAD67 (1:1000; Sigma Millipore, catalog \#MAB5406), rabbit anti-NeuN (1:500; Sigma Millipore, catalog \#ABN78), rabbit Neuro-Chrom pan neuronal marker (1:1000; Sigma Millipore, catalog \#ABN2300), rabbit anti-NPY (1:1000; Peninsula Labs, catalog \#T-4070), guinea pig anti-GlyT2 (1:2000; Synaptic Systems, cata$\log \# 272-004)$, or mouse anti-bNOS (1:1000; Sigma Millipore, catalog \#N2280). On the following day, sections were rinsed in PBS and incubated in AlexaFluor-647-tagged goat anti-mouse IgG or AlexaFluor750-tagged goat anti-rabbit IgG (1:100; Thermo Fisher Scientific, catalog \#A21235 and \#A21039), or AlexaFluor-647-tagged donkey anti-mouse IgG, AlexaFluor-647-tagged donkey anti-rabbit IgG, or AlexaFluor-594tagged goat anti-guinea pig IgG (1:500; Thermo Fisher Scientific, catalog \#A-21202, \#A-21206, and \#A-11076) for 1.0-1.5 h at room temperature. Sections were then mounted on Superfrost Plus microscope slides (Thermo Fisher Scientific, catalog \#12-550-15) and coverslipped using Fluoromount-G (SouthernBiotech, catalog \#0100-01) or DPX (Sigma Millipore, catalog \#06522). Images were collected using a $1.30 \mathrm{NA} 40 \times$ oil-immersion objective or a 1.40 NA $63 \times$ oil-immersion objective on a Leica TCS SP8 laser scanning confocal microscope.

\section{Antibody validation}

All antibodies used in this study, except anti-NPY, have been previously validated in the IC. To label GABAergic neurons, we used the mouse monoclonal anti-GAD67 antibody (Sigma Millipore, catalog \#MAB5406). This antibody was raised against the $67 \mathrm{kDa}$ isoform of glutamic acid decarboxylase (GAD). The manufacturer reports that Western blot analysis showed no cross-reactivity with the $65 \mathrm{kDa}$ isoform of GAD (GAD65). This antibody has been used in several studies to identify GABAergic neurons in the IC (Ito et al., 2009; Mellott et al., 2014; Beebe et al., 2016; Goyer et al., 2019). To identify neurons, we performed anti-NeuN staining with a rabbit polyclonal antibody (Sigma Millipore, catalog \#ABN78). The manufacturer reports that anti-NeuN specifically recognizes the DNA-binding, neuron-specific protein NeuN. Previous studies used this antibody to identify neurons in the IC (Foster et al., 2014; Mellott et al., 2014; Beebe et al., 2016; Goyer et al., 2019). To define the borders of IC subdivisions, we performed a double staining using anti-GAD67 and anti-GlyT2 antibodies (Buentello et al., 2015). Guinea pig anti-GlyT2 
antibody (Synaptic Systems, catalog \#272004) was used to identify glycine transporter 2, which is localized in axons. We also immunostained with anti-brain nitric oxide synthase (bNOS) to define the borders of the IC subdivisions (Sigma Millipore, catalog \#N2280). This antibody was raised against the IgG1 isotype from the NOS-B1 hybridoma. The manufacturer reports that anti-bNOS reacts specifically with nitric oxide synthase (NOS), derived from brain (bNOS, 150-160 kDa). Previous studies used anti-bNOS to delineate the borders of IC subdivisions in guinea pig and mouse (Coote and Rees, 2008; Keesom et al., 2018). Finally, to label NPY, we used the polyclonal rabbit anti-NPY antibody (Peninsula Laboratories, catalog \#T-4070). Pretreatment with antiserum containing NPY peptide abolished all staining, reinforcing the high specificity of this antibody (Rowan et al., 1993). This antibody has been successfully used in cortex (Rose et al., 2009; Milstein et al., 2015) and hippocampus (Ledoux et al., 2009).

\section{Analysis of GAD67 staining}

Analysis of GAD67 immunostaining was performed as previously reported (Goyer et al., 2019). Images from representative sections of the IC ( $n=2$ male P74 mice, three sections per mouse: one caudal, one medial, and one rostral) were collected at $2 \mu \mathrm{m}$ depth intervals with a 1.30 NA $40 \times$ oil-immersion objective on a TCS SP8 laser scanning confocal microscope (Leica Microsystems). Images were analyzed using LAS $\mathrm{X}$ software (version 3.3.0, Leica Microsystems). As previously reported, anti-GAD67 antibody did not penetrate the entire depth of the tissue sections (Beebe et al., 2016; Goyer et al., 2019). For this reason, the analysis was restricted to the top 10-12 $\mu \mathrm{m}$ of each section, where the antibody fully penetrated. Within this region, we manually marked every $\mathrm{hrGFP}^{+}$ cell body in the left IC. We analyzed the green (hrGFP) and red (Alexa647) channels separately, so that labeling in one channel did not influence analysis of the other channel. After the hrGFP cells were marked, the red and green color channels were merged; and in every instance where a cell body contained markers for hrGFP, we evaluated whether GAD67 was also present. The number of double-labeled cells was compared with the total number of $\mathrm{hrGFP}^{+}$neurons to determine the percentage of hrGFP ${ }^{+}$neurons that were $\mathrm{GAD}^{+} 7^{+}$as well.

\section{Analysis of NeuN staining with design-based stereology}

We used a design-based stereology approach to estimate the numbers of $\mathrm{hrGFP}^{+}$and NeuN ${ }^{+}$neurons in brain sections that were stained with anti-NeuN antibody. This approach has been previously described and validated in the IC (Goyer et al., 2019). In brief, we collected systematic random samples using a virtual grid of $370 \mu \mathrm{m} \times 370 \mu \mathrm{m}$ squares that was overlaid on the IC section. The starting coordinates for the grid were set using the Mersenne Twister random number generator in Igor Pro 7 or 8 (WaveMetrics). We then collected images of the coordinates determined by the top left intersection of each grid square that fell over the IC. Each image consisted of a $184 \mu \mathrm{m} \times 184 \mu \mathrm{m} z$ stack collected at $1 \mu \mathrm{m}$ depth intervals with a $1.40 \mathrm{NA} 63 \times$ oil immersion objective on a Leica TCS SP8 confocal microscope (Leica Microsystems). Eight to 20 images were collected per slice. Three slices (caudal, middle, and rostral) were analyzed per mouse $(n=2)$. Using Neurolucida 360 (MBF Bioscience), we counted neurons using the optical fractionator approach (West et al., 1991). In this approach, we defined guard zones as $\geq 2-\mu \mathrm{m}-$ thick regions at the top and bottom of the slice and excluded these from subsequent analysis. Neurons between the guard zones, within a $15-\mu \mathrm{m}-$ thick region at the center of the slice, were counted by making a single mark at the top of each cell. Cells crossing the right and top borders of the image stack were counted, whereas those crossing the left and bottom borders were not. The red (NeuN) and green (hrGFP) color channels were analyzed separately, so that labeling in one channel did not influence analysis of the other. In all neurons counted, $\mathrm{hrGFP}^{+}$cells were also $\mathrm{NeuN}^{+}$(1097 of 1097 cells). The total number of double-labeled $\left(\mathrm{hrGFP}^{+} / \mathrm{NeuN}^{+}\right)$cells was then compared with the total number of $\mathrm{NeuN}^{+}$cells. We also collected tile scan images of each IC section analyzed using a $10 \times$ objective and used GAD67 and GlyT2 staining to determine the border separating the ICc from the IC shell (ICs) regions. The $63 \times z$ stacks were aligned to these tile scans using Adobe Photoshop, and counted neurons were assigned to the ICc or ICs.

\section{Analysis of GAD67 staining in Npy1r ${ }^{\text {cre }}$ mice with design-based stereology}

To evaluate the neurotransmitter content of neurons that express the $\mathrm{Y}_{1} \mathrm{R}$, we performed anti-GAD67 staining on brain slices of $N p y 1 r^{c r e} \mathrm{x}$ Ail4 mice. We then used design-based stereology to quantify the proportion of tdTomato $^{+}$neurons that colabeled with GAD67. This analysis was performed as described above, except that since anti-GAD67 antibody does not penetrate the entire depth of tissue sections (Beebe et al., 2016; Goyer et al., 2019), the analysis was restricted to depths at which clear anti-GAD67 immunostaining was present.

\section{Brain slice preparation}

To characterize the intrinsic physiology of NPY neurons and the effect of NPY on neurons in the IC, whole-cell patch-clamp recordings were performed in NPY-hrGFP (van den Pol et al., 2009) and Npylr ${ }^{\text {cre }}$ mice (Padilla et al., 2016). Both males ( $n=22$ for NPY-hrGFP mice, $n=12$ for Npy $1 r^{\text {cre }} \mathrm{x}$ Ai14 mice) and females $(n=33$ for NPY-hrGFP mice, $n=12$ for Npy1r ${ }^{c r e}$ x Ail4 mice) aged P22-P50 were used. No differences were observed between animals from different sexes (linear regression, $p>0.05)$. Mice were deeply anesthetized with isoflurane and then rapidly decapitated. The brain was removed, and the IC was dissected in $\sim 34^{\circ} \mathrm{C}$ ACSF containing the following (in $\mathrm{mM}$ ): $125 \mathrm{NaCl}, 12.5$ glucose, $25 \mathrm{NaHCO}_{3}, 3 \mathrm{KCl}, 1.25 \mathrm{NaH}_{2} \mathrm{PO}_{4}, 1.5 \mathrm{CaCl}_{2}, 1 \mathrm{MgSO}_{4}, 3$ sodium pyruvate, and $0.40 \mathrm{~L}$-ascorbic acid, bubbled to a $\mathrm{pH}$ of 7.4 with $5 \% \mathrm{CO}_{2}$ in $95 \% \mathrm{O}_{2}$. Coronal sections of the IC $(200 \mu \mathrm{m})$ were cut in $\sim 34^{\circ} \mathrm{C}$ ACSF with a vibrating microtome (VT1200S, Leica Biosystems) and incubated at $34^{\circ} \mathrm{C}$ for $30 \mathrm{~min}$ in ACSF bubbled with $5 \% \mathrm{CO}_{2}$ in $95 \% \mathrm{O}_{2}$. After incubation, slices were placed at room temperature for at least $30 \mathrm{~min}$ before being transferred to the recording chamber. All chemicals were obtained from Thermo Fisher Scientific unless otherwise noted.

\section{Electrophysiological recordings}

For recordings, slices were placed into a chamber that was continuously perfused at $\sim 2 \mathrm{ml} / \mathrm{min}$ with $34^{\circ} \mathrm{C}$ oxygenated ACSF. NPY-hrGFP neurons and $\mathrm{Y}_{1} \mathrm{R}$-tdTomato neurons in IC slices were identified with epifluorescence using a Nikon FN1 microscope. Whole-cell current-clamp recordings were performed with a BVC-700A patch-clamp amplifier (Dagan). Data were low-pass filtered at $10 \mathrm{kHz}$, sampled at $50 \mathrm{kHz}$ with a National Instruments PCIe-6343 data acquisition board, and acquired using custom software written in IgorPro.

Recording pipettes were pulled from borosilicate glass pipettes (outer diameter $1.5 \mathrm{~mm}$, inner diameter $0.86 \mathrm{~mm}$, catalog \#BF150-86-10, Sutter Instrument) using a P-1000 microelectrode puller (Sutter Instrument). Pipettes were filled with an internal solution containing the following (in $\mathrm{mM}$ ): $115 \mathrm{~K}$-gluconate, $7.73 \mathrm{KCl}, 0.5 \mathrm{EGTA}, 10 \mathrm{HEPES}, 10 \mathrm{Na}_{2}$-phosphocreatine, 4 MgATP, $0.3 \mathrm{NaGTP}$, supplemented with $0.1 \%$ biocytin (w/v), $\mathrm{pH}$ adjusted to 7.3 with $\mathrm{KOH}$ and osmolality to $290 \mathrm{mmol} / \mathrm{kg}$ with sucrose. Pipettes with resistances of 3.0-4.8 $\mathrm{M} \Omega$ when filled with internal solution were used for recordings.

Input resistance was assessed by applying a series of $100 \mathrm{~ms}$ current steps that hyperpolarized the membrane potential from just below rest to $<-100 \mathrm{mV}$. For each step response, the peak and steady-state voltage changes were measured, and these values were used to generate voltage versus current plots from which the peak $\left(R_{p k}\right)$ and steady-state $\left(R_{s s}\right)$ input resistances were calculated based on the slope of a linear regression. Membrane time constant was determined by applying $50,300 \mathrm{~ms}$ current steps that hyperpolarized the membrane potential by $2-4 \mathrm{mV}$ and taking the median of the time constants obtained by fitting an exponential function to each response. All membrane potential values were corrected for the liquid junction potential $(-11 \mathrm{mV})$. Capacitance and series resistance were compensated using bridge balance. Recordings with series resistance $>20 \mathrm{M} \Omega$ or changes in series resistance $>15 \%$ during the recording period were discarded.

To test whether NPY neurons fire in the presence of synaptic blockers $(n=8)$, we blocked synaptic inputs using $50 \mu \mathrm{M}$ D-AP5 (NMDA receptor antagonist, Hello Bio, catalog \#HB0225) and $10 \mu \mathrm{M} \mathrm{NBQX}$ disodium salt (AMPA receptor antagonist, Hello Bio, catalog \#HB0443). All drugs were diluted in standard ACSF. 
To evaluate the effects of $\mathrm{Y}_{1} \mathrm{R}$ activation, $\mathrm{Y}_{1} \mathrm{R}$ tdTomato neurons were targeted for recordings. Since most NPY neurons spontaneously fire $(n=87$ of 121 cells), we included $1 \mu \mathrm{M}$ TTX (Hello Bio, catalog \#HB1035) in the ACSF in this experiment to block action potential mediated release of NPY. In current-clamp mode, $Y_{1} R$ neurons were held for $10 \mathrm{~min}$ before starting the control period recording to limit possible effects of intracellular dialysis. During these $10 \mathrm{~min}, R_{p k}, R_{s s}$ and membrane time constant were collected to monitor the health of the neuron. At the start of the 5 min control period, we initiated a protocol that recorded the resting membrane potential $\left(V_{\text {rest }}\right)$ every $10 \mathrm{~s}$. Only neurons with a stable $V_{\text {rest }}$, defined as $V_{\text {rest }}$ drift $<3 \mathrm{mV}$, were included in the analysis. We then applied $500 \mathrm{~nm}\left[\mathrm{Leu}^{31}\right.$, Pro ${ }^{34}$ NPY (Tocris Bioscience, catalog \#1176), a high-affinity $\mathrm{Y}_{1} \mathrm{R}$ agonist. We used $500 \mathrm{~nm}\left[\mathrm{Leu}^{31}, \mathrm{Pro}^{34}\right]$-NPY based on previous studies performed in the hypothalamus (Roa and Herbison, 2012) and hippocampus (Giesbrecht et al., 2010). [ $\mathrm{Leu}^{31}, \mathrm{Pro}^{34}$ ]-NPY was diluted in $10 \mathrm{ml}$ of ACSF and bath-applied for $\sim 5 \mathrm{~min}$. The recording was then continued for a 20-50 min washout period. Only neurons that had a washout period over 20 min were included in the analysis. Control experiments followed the same approach, using a vehicle solution (normal ACSF).

To analyze the effect of $\left[\mathrm{Leu}^{31}, \mathrm{Pro}^{34}\right]$-NPY on the $V_{\text {rest }}$ of IC neurons, we compared $V_{\text {rest }}$ during the final 2 min of the baseline period with the initial 2 min of the drug application period (see Fig. $8 A, B$, gray bars). The drug application period started $90 \mathrm{~s}$ after the end of the control period to allow time for the drug solution to reach the recording chamber. A cell was considered sensitive to $\left[\right.$ Leu $^{31}$, Pro $\left.^{34}\right]$-NPY if it exhibited a $>2 \mathrm{mV}$ change in $V_{\text {rest }}$ compared with baseline.

To extend the results from the bath application of $\left[\mathrm{Leu}^{31}, \mathrm{Pro}^{34}\right]-\mathrm{NPY}$, in a subsequent experiment, $\left[\mathrm{Leu}^{31}\right.$,

$\left.\mathrm{Pro}^{34}\right]$-NPY was delivered using a puffer pipette. The puffer pipette was placed close to the recorded cell, allowing the drug to reach $Y_{1} R$ neurons faster than was possible with the bath application. The puffer pipette was filled with $1 \mu \mathrm{M}\left[\mathrm{Leu}^{31}\right.$, Pro $\left.{ }^{34}\right]$-NPY diluted in ACSF. In current- clamp mode, $R_{p k}, R_{s s}$, and membrane time constant were recorded for each cell. Next, we recorded $30 \mathrm{~s}$ of baseline and then puff-applied [Leu ${ }^{31}, \mathrm{Pro}^{34}$ ]NPY for $60 \mathrm{~s}$ ( $n=6$ animals, 9 cells) (Acuna-Goycolea and van den Pol, 2005). For control experiments ( $n=5$ animals, 7 cells), the puffer pipette was filled with ACSF and the position of the pipette and duration of the puff were identical to the drug group. To analyze the effect of puffing $\left[\mathrm{Leu}^{31}, \mathrm{Pro}^{34}\right]$-NPY, we compared the mean $V_{\text {rest }}$ during the final $20 \mathrm{~s}$ of the baseline period with the mean $V_{\text {rest }}$ during the middle $20 \mathrm{~s}$ of the puff application (20-40 s after puff onset).

Post hoc reconstructions of morphology and morphology analysis Neurons were filled with biocytin via the recording pipette. After the recording, an outside-out patch was formed, by slowly removing the pipette, to allow the cell membrane to reseal. Brain slices were then fixed overnight in $4 \% \mathrm{PFA}$ in $0.1 \mathrm{M} \mathrm{PB}, \mathrm{pH} 7.4$, and moved to PBS the next day. Slices were stored at $4^{\circ} \mathrm{C}$ for up to 3 weeks and then stained using fluorescent biocytin-streptavidin histochemistry. For this, slices were washed in PBS 3 times for $10 \mathrm{~min}$, the membrane was permeabilized in $0.2 \%$ Triton X-100 in PBS for $2 \mathrm{~h}$, washed $3 \times 10 \mathrm{~min}$ in PBS, and stained at $4^{\circ} \mathrm{C}$ for $24 \mathrm{~h}$ with streptavidin-AlexaFluor-647 (1:1000). The next day, slices were washed 3 $\times 10$ min with PBS, postfixed in 4\% PFA in $0.1 \mathrm{M} \mathrm{PB}, \mathrm{pH} 7.4$, for $1 \mathrm{~h}$, and then washed $3 \times 10 \mathrm{~min}$ with PBS. Slices were then mounted on Superfrost Plus microscope slides in anti-fade media (Fluoromount-G). Images were obtained with a TCS SP8 laser scanning confocal microscope (Leica Microsystems) using a 1.40 NA $63 \times$ oil-immersion objective. $z$ stack tile scans were performed to image the soma and dendritic arbor. Image stacks were imported into Neurolucida 360 (MBF Bioscience), where $3 \mathrm{D}$ reconstructions and quantitative analyses of neuronal morphology were performed.
Table 1. hrGFP $^{+}$neurons express NPY ${ }^{a}$

\begin{tabular}{llrrrl}
\hline Animal & Slice $(n)$ & $\begin{array}{c}\text { No. of } \\
\text { hrGFP }\end{array}$ & $\begin{array}{c}\text { No. of } \\
\mathrm{NPY}^{+}\end{array}$ & $\begin{array}{l}\text { No. of hrGFP } \\
\mathrm{NPY}^{-}\end{array}$ & $\begin{array}{l}\% \mathrm{hrGFP}^{+} \\
\mathrm{NPY}^{+}\end{array}$ \\
\hline P58 male 1 & 1 (caudal) & 464 & 436 & 28 & 94.0 \\
& 2 (middle) & 563 & 537 & 26 & 95.4 \\
& 3 (rostral) & 567 & 537 & 30 & 94.5 \\
& Total & 1594 & 1510 & 84 & 94.7 \\
P58 male 2 & 1 (caudal) & 496 & 485 & 11 & 97.8 \\
& 2 (middle) & 602 & 555 & 47 & 92.2 \\
& 3 (rostral) & 514 & 486 & 28 & 94.6 \\
& Total & 1612 & 1526 & 86 & 94.7 \\
Grand total & & 3206 & 3036 & 170 & 94.7 \\
Average across 2 mice & & & & 94.7 \\
\hline
\end{tabular}

${ }^{a}$ Across 2 mice, an average of $94.7 \%$ of hrGFP ${ }^{+}$neurons were labeled with an antibody against NPY.

\section{Retrograde tracing}

In 7 NPY-hrGFP mice, we used red fluorescent RetroBeads (RBs) to evaluate whether NPY neurons participate in the IC-MG projection ("red beads," Lumafluor; 1:2 dilution). Surgeries were performed on males and females, aged P22-P28, using standard aseptic techniques. Mice were deeply anesthetized with isoflurane (2\%-3\%) and their body temperature maintained with a homeothermic heating pad. The analgesic carprofen ( $5 \mathrm{mg} / \mathrm{kg}$, CarproJect, Henry Schein Animal Health) was injected subcutaneously. The scalp was shaved, and a rostrocaudal incision was made along the midline to expose the skull. A unilateral craniotomy was performed, using a micromotor drill (K.1050, Foredom Electric) with a $0.5 \mathrm{~mm}$ burr (Fine Science Tools). The coordinates were defined relative to the bregma suture, with injection depths relative to the surface of the brain. Injections were made in one penetration: $3200 \mu \mathrm{m}$ caudal, $1900 \mu \mathrm{m}$ lateral, and $2750 \mu \mathrm{m}$ deep.

RBs were injected with a NanoJect III nanoliter injector (Drummond Scientific) connected to a MP-285 micromanipulator (Sutter Instruments). 

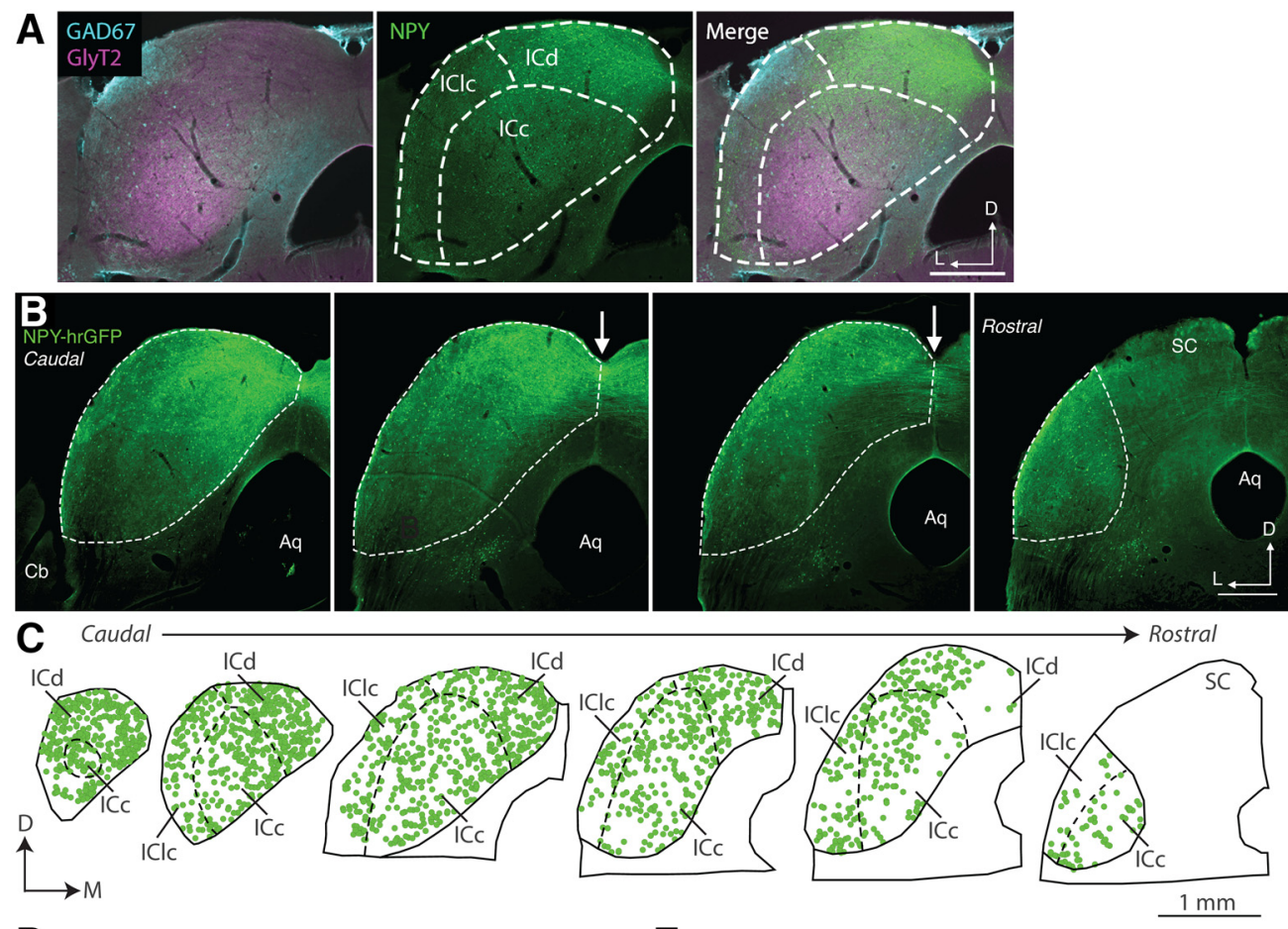

D

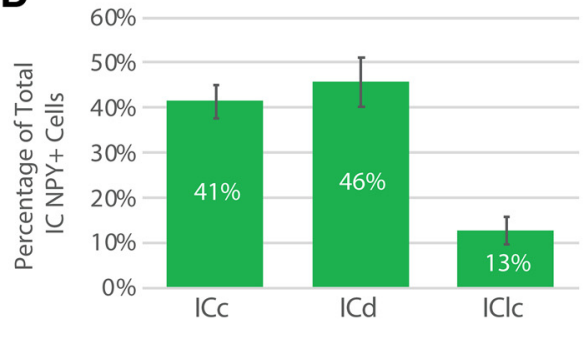

E

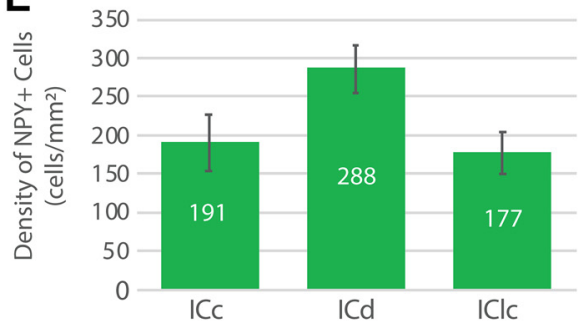

Figure 2. NPY neurons are distributed throughout the IC. A, Micrographs of a single coronal section through the IC of an NPY-hrGFP mouse stained for GAD67 (cyan) and GlyT2 (magenta). The ICc has relatively more staining for GlyT2 ${ }^{+}$terminals compared with cortical subdivisions. Increased GAD67 staining separates the IClc from the ICd, which has relatively less staining for GAD67. Top, Dorsal. Left, Lateral. Scale bar, $500 \mu \mathrm{m}$. B , Micrographs of a series of coronal sections through the IC of an NPY-hrGFP mouse. NPY ${ }^{+}$cells (green) were observed in all sections through the IC. White arrows indicate NPY ${ }^{+}$axons crossing the IC commissure. Top, Dorsal. Left, Lateral. Aq, Cerebral aqueduct; Cb, cerebellum; SC, superior colliculus. Scale bar, 500 um. C, A plot through the three main subdivisions of the IC showing the distribution of NPY cells. Each green circle represents one NPY neuron. Sections are arranged from caudal to rostral. Top, Dorsal. Right, Medial. Scale bar, $1 \mathrm{~mm}$. D, Bar graph represents the mean \pm SD of the proportion of the NPY ${ }^{+}$population present in each of the main IC subdivisions. The ICd contained the highest proportion of the NPY ${ }^{+}$population, wheres the IClc contained the lowest proportion. $\boldsymbol{E}$, Bar graph represents the mean \pm SD of the density of NPY ${ }^{+}$cells in each of the IC subdivisions. The $\mathrm{ICd}$ contained the highest density of NPY${ }^{+}$cells, and the IClc contained the lowest density.

Glass injection pipettes were pulled using $1.14 \mathrm{~mm}$ outer diameter, 0.53 $\mathrm{mm}$ inner diameter capillary glass (catalog \#3-000-203-G/X, Drummond Scientific) with a P-1000 microelectrode puller (Sutter Instrument). The injector tip was cut and back-filled with mineral oil and then front-filled with the RBs. After the injections were complete, the scalp was sutured, using Ethilon 6-0 (0.7 metric) nylon sutures (Ethicon), and the incision was treated with $0.5 \mathrm{ml} 2 \%$ lidocaine hydrochloride jelly (Akorn). After recovery from anesthesia, mice were returned to the vivarium and were monitored daily until the wound healed. After 5-7 d, mice were perfused and projections were analyzed.

\section{Subdivisions of the IC}

We used two different approaches to define the borders between the ICc, ICd, and IClc. For the stereology experiments and for the analysis of the distribution of NPY neurons, subdivision borders were defined using a combination of GlyT2 and GAD67 staining (Buentello et al., 2015). To identify the subdivisions in which reconstructed neurons were located, we used bNOS staining (Coote and Rees, 2008). Both methods have been previously validated in the IC, and we did not observe any obvious difference between the subdivision borders obtained with these approaches.

\section{Statistics}

All statistical analyses were performed in Igor Pro. Data are presented as mean $\pm \mathrm{SD}$. The statistical tests used for each experiment are indicated in Results. Results were considered significant when $p<0.05$. For comparing the intrinsic physiology of sustained and adapting $\mathrm{Y}_{1} \mathrm{R}$-expressing neurons, the significance level $(\alpha)$ was adjusted with the Bonferroni correction to account for the multiple comparisons performed (adjusted $\alpha=0.05 / 8=0.00625$; see Fig. $7 G$ ).

\section{Results}

NPY neurons are distributed throughout the main subdivisions of the IC

To target experiments to NPY-expressing neurons, we obtained the NPY-hrGFP mouse line, in which hrGFP expression is driven by the NPY promoter (van den Pol et al., 2009). We performed immunostaining against the NPY peptide to determine whether this mouse line selectively labels NPY-expressing neurons in the IC. In brain slices from 2 P58 males, we counted $\mathrm{hrGFP}^{+}$neurons in one caudal, one middle, and one rostral section of the IC per animal $\left(n=3206 \mathrm{hrGFP}^{+}\right.$neurons; Fig. 
Table 2. NPY neurons are GABAergic ${ }^{a}$

\begin{tabular}{llcccc}
\hline Animal & Slice $(n)$ & No. of hrGFP & No. of GAD67 & No. of hrGFP GAD67 $^{+}$ & \% $^{+}$ \\
\hline P74 male 1 & 1 (caudal) & 456 & 450 & 6 & 98.7 \\
& 2 (middle) & 387 & 380 & 7 & 98.2 \\
& 3 (rostral) & 328 & 322 & 6 & 98.2 \\
P74 male 2 & Total & 1171 & 1152 & 19 & 98.4 \\
& 1 (caudal) & 468 & 462 & 6 & 98.7 \\
& 2 (middle) & 346 & 337 & 9 & 97.4 \\
Grand total & 3 (rostral) & 688 & 682 & 6 & 99.1 \\
Average across 2 mice & Total & 1502 & 1481 & 21 & 98.6 \\
\hline
\end{tabular}

${ }^{a}$ Across 2 mice, an average of $98.5 \%$ of hrGFP ${ }^{+}$neurons were labeled with an antibody against GAD67.

$1 A-C)$. We then evaluated whether each $\mathrm{hrGFP}^{+}$neuron colabeled with anti-NPY. We found that $94.7 \%$ of $\mathrm{hrGFP}^{+}$neurons were $\mathrm{NPY}^{+}$, indicating that the NPY-hrGFP mouse line provides highly selective labeling of NPY-expressing neurons $\left(n=3036 \mathrm{hrGFP}^{+} / \mathrm{NPY}^{+}\right.$neurons and $170 \mathrm{hrGFP}^{+} / \mathrm{NPY}^{-}$ neurons; Table 1).

We observed hrGFP-labeled cells throughout the rostrocaudal extent of the auditory midbrain in NPY-hrGFP mice, including a presence across IC subdivisions and in the nucleus of the brachium of the IC and the intercollicular tegmentum. We observed virtually no NPY neurons in auditory brainstem nuclei outside the midbrain. Within the auditory midbrain, IC subdivisions were identified by the pattern of GAD67 and GlyT2 immunofluorescence (Fig. 2A), and $\sim 95 \%$ of the NPY neurons were present in the three main subdivisions of the IC. Figure $2 B$ shows the distribution of NPY neurons (green) through the rostrocaudal extent of the three major IC subdivisions. We plotted each individual NPY neuron in the IC in 3 cases (Fig. 2C). The ICd contained the most NPY cells ( $46 \pm 6 \%$ of the IC NPY population), whereas the IClc contained the fewest (13 $\pm 3 \%$; Fig. $2 D$ ). The ICd also had the highest average density of NPY neurons $\left(288 \pm 32\right.$ cells per $\left.\mathrm{mm}^{2}\right)$, whereas the IClc had the lowest average density $\left(177 \pm 28\right.$ cells per $\mathrm{mm}^{2}$; Fig. $\left.2 E\right)$.

\section{NPY neurons represent one-third of the GABAergic neurons in the IC}

NPY neurons are nearly exclusively GABAergic in the cerebral cortex (Karagiannis et al., 2009), hippocampus (Jinno and Kosaka, 2003), and hypothalamus (Henry et al., 2015; Marshall et al., 2017). To investigate the neurotransmitter content of NPY neurons in the IC, we performed immunostaining against GAD67, an enzyme essential for GABA synthesis. Using brain slices from 2 P74 males (Fig. $1 D-F$ ), we counted $\mathrm{hrGFP}^{+}$neurons in one caudal, one middle, and one rostral section of the IC per animal ( $n=2673 \mathrm{hrGFP}^{+}$neurons). We then determined whether each hrGFP $^{+}$neuron colabeled with anti-GAD67. Of the $2673 \mathrm{hrGFP}^{+}$neurons counted, $98.5 \%$ were $\mathrm{GAD}^{+}$ $\left(n=2633 \mathrm{hrGFP}^{+} / \mathrm{GAD}^{+} 7^{+}\right.$neurons and $40 \mathrm{hrGFP}^{+} / \mathrm{GAD}^{-} 7^{-}$ neurons; Table 2). We suspect that the absence of GAD67 immunostaining in $40 \mathrm{hrGFP}^{+}$neurons was due to rare cases of poor antibody penetration or nonspecific expression of hrGFP. Thus, these data indicate that all, or nearly all, NPY neurons in the IC are GABAergic.

Next, we used design-based stereology to determine the percentage of neurons in the IC that express NPY. For this experiment, we analyzed the ICc and IC shell (ICs = ICd plus IClc) separately, defining the borders between these subdivisions according to the pattern of GAD67 and GlyT2 immunoreactivity
Table 3. NPY neurons represent $7.6 \%$ of neurons in the ICC and $7.8 \%$ of neurons in the IC shell ${ }^{a}$

\begin{tabular}{llcll}
\hline Coronal slice & P58 Male 1 & P58 Male 2 & Per slice pane & Grand average \\
\hline ICC & & & & \\
Caudal & & & & \\
& $(6.0 \%$ & $9.4 \%$ & $8.2 \%$ & \\
& $(60 / 755)$ & $(15 / 159)$ & $(75 / 914)$ & \\
Medial & 7 samples & 4 samples & 11 samples & \\
& $7.9 \%$ & $7.0 \%$ & $7.4 \%$ & \\
& $(84 / 1070)$ & $(90 / 1278)$ & $(174 / 2348)$ & \\
Rostral & 11 samples & 9 samples & 20 samples & \\
& $7.7 \%$ & $7.2 \%$ & $7.5 \%$ & \\
& $(77 / 999)$ & $(68 / 942)$ & $(145 / 1941)$ & \\
Per mouse & 10 samples & 10 samples & 20 samples & \\
& $7.8 \%$ & $7.3 \%$ & & \\
& $(221 / 2824)$ & $(173 / 2379)$ & & $(394 / 5203)$
\end{tabular}

IC shell

\begin{tabular}{|c|c|c|c|c|}
\hline \multirow[t]{2}{*}{ Caudal } & $\begin{array}{l}8.6 \% \\
(140 / 1620)\end{array}$ & $\begin{array}{c}6.3 \% \\
(29 / 460)\end{array}$ & $\begin{array}{l}8.1 \% \\
(169 / 2080)\end{array}$ & \\
\hline & 12 samples & 7 samples & 19 samples & \\
\hline \multirow[t]{2}{*}{ Medial } & $\begin{array}{l}6.9 \% \\
(128 / 1849)\end{array}$ & $\begin{array}{l}10.4 \% \\
(76 / 728)\end{array}$ & $\begin{array}{l}7.9 \% \\
(204 / 2577)\end{array}$ & \\
\hline & 11 samples & 8 samples & 19 samples & \\
\hline Rostral & $\begin{array}{l}8.3 \% \\
(55 / 668)\end{array}$ & $\begin{array}{l}6.7 \% \\
(77 / 1155)\end{array}$ & $\begin{array}{l}7.2 \% \\
(132 / 1823)\end{array}$ & \\
\hline Per mouse & $\begin{array}{l}7.8 \% \\
(323 / 4137)\end{array}$ & $\begin{array}{c}7.8 \% \\
(182 / 2343)\end{array}$ & & $\begin{array}{l}7.8 \% \\
(505 / 6480)\end{array}$ \\
\hline
\end{tabular}

${ }^{a}$ Stereological analysis of the percentage of neurons $\left(\mathrm{NeuN}^{+}\right)$in the $\mathrm{ICC}$ and $\mathrm{IC}$ shell $(\mathrm{ICd}+\mathrm{IClc})$ that expressed hrGFP in NPY-hrGFP mice.

(Buentello et al., 2015). Sections from 2 P54 male NPY-hrGFP mice were stained with anti-NeuN, a neuron-selective antibody previously shown to label most or all neurons in the IC (Fig. 1G-I) (Mellott et al., 2014; Beebe et al., 2016). Three sections per mouse were analyzed: one caudal, one middle, and one rostral. To ensure unbiased counting of neurons, we applied the optical fractionator method (see Materials and Methods). Sections adjacent to the anti-NeuN sections were immunostained with anti-GAD67 and anti-GlyT2, and IC subdivision boundaries were determined by comparing these with the adjacent anti-NeuN-stained sections. This analysis showed that NPY neurons represented $7.6 \%$ of neurons in the ICc and $7.8 \%$ in the ICs (Table 3). Since previous studies indicate that $20 \%-27 \%$ of IC neurons are GABAergic (Oliver et al., 1994; Merchán et al., 2005; Beebe et al., 2016), our results suggest that NPY-expressing neurons account for approximately one-third (27\%-39\%) of GABAergic neurons in the IC. 
A
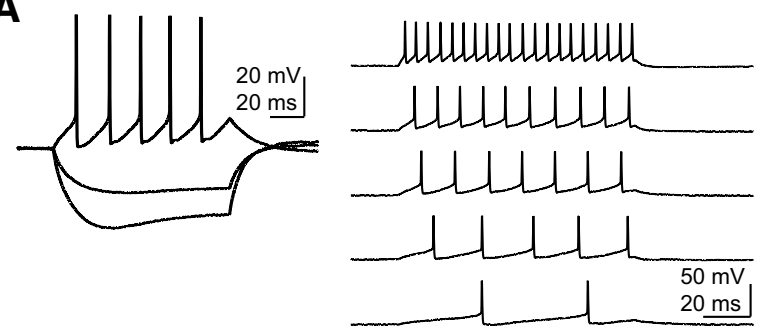

C
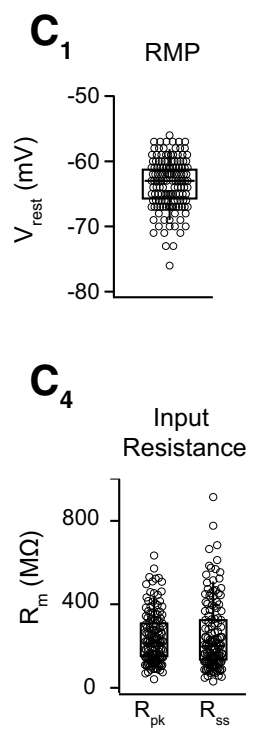

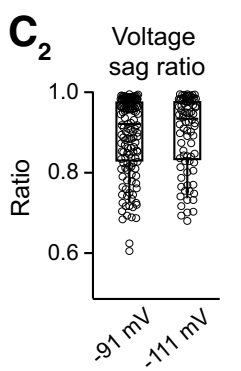

$\mathrm{C}_{5}$

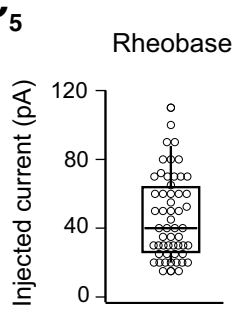

B

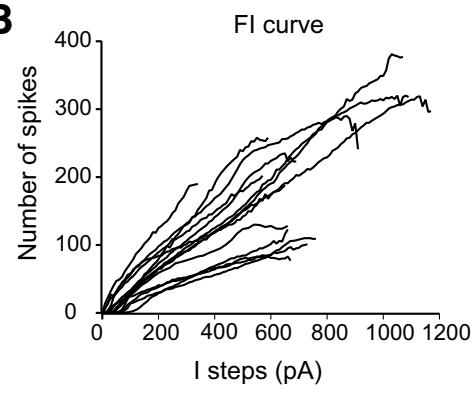

D

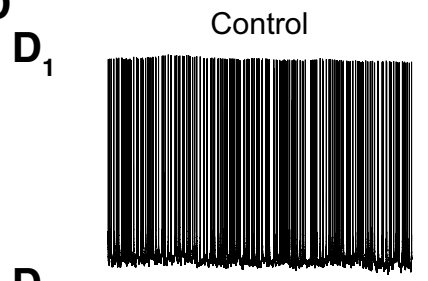

$\mathbf{D}_{\mathbf{2}}+50 \mu \mathrm{M}$ AP5 \& $10 \mu \mathrm{M}$ NBQX

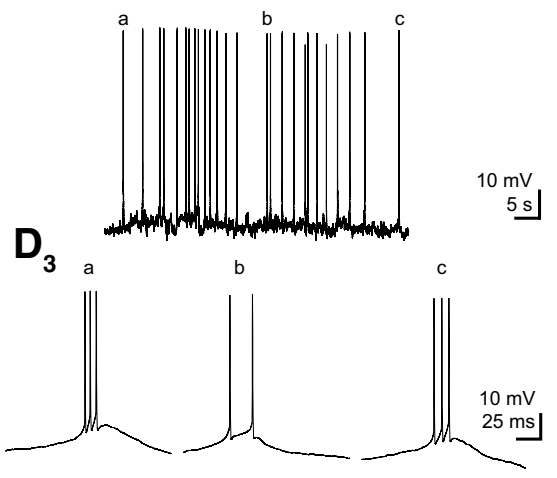

Figure 3. NPY neurons have a sustained firing pattern and most fire spontaneously. $A$, NPY neurons exhibited a sustained firing pattern in response to depolarizing current steps and a moderate voltage sag in response to hyperpolarizing current steps. $\boldsymbol{B}$, Firing versus input analysis showed a linear input-output response, with increasing firing rates in response to increasingly large depolarizing current steps. Example Fl curves from 15 neurons are shown. $\boldsymbol{C}$, NPY neurons exhibited moderate intrinsic physiological properties. $\boldsymbol{C}_{1}$, Resting membrane potential. $\boldsymbol{C}_{2}$, Voltage sag ratio. $\boldsymbol{C}_{3}$, Membrane time constant. $\boldsymbol{C}_{4}$ Input resistance. $\boldsymbol{C}_{5}$, Rheobase. $\boldsymbol{C}_{6}$, SFA ratio. Boxplots represent median, $25^{\text {th }}$ and $75^{\text {th }}$ percentile (box), and $9^{\text {th }}$ and $91^{\text {st }}$ percentile (whiskers). $\boldsymbol{D}$, Most NPY neurons spontaneously fired at rest $\left(\boldsymbol{D}_{1}\right)$, and spontaneous firing persisted in 7 of 8 cells in the presence of AMPA and NMDA receptor antagonists $\left(\boldsymbol{D}_{2}, 10 \mu \mathrm{m}\right.$ NBQX and $50 \mu \mathrm{m}$ DAP5). $\boldsymbol{D}_{3}$, An expanded view of three spiking events from $\boldsymbol{D}_{2}$ shows that, in the presence of the antagonists, action potentials appeared to rise smoothly from baseline.

Together, the above data show that NPY is expressed in a population of GABAergic neurons in the IC, but do not indicate whether this population represents one or several neuron types. To test this, we next analyzed the physiological and morphologic properties of NPY neurons.

\section{Most NPY neurons exhibit a sustained firing pattern and have a propensity to spontaneously fire}

In acute brain slices, we targeted whole-cell current-clamp recordings to NPY-hrGFP neurons in the ICc, ICd, and IClc. The location of the recording was noted at the end of the recording and confirmed during retrieval of neuronal morphology (morphology was recovered from 49 of 146 recorded neurons). Because the intrinsic physiology of NPY neurons did not differ across IC subdivisions, neurons from ICc, ICd, and IClc are lumped together here. Most NPY neurons presented moderate voltage sag in response to hyperpolarizing current steps (Fig. $3 \mathrm{~A}$, left) and a sustained firing pattern in response to depolarizing current steps (Fig. 3A, right). Firing versus current functions were determined with $1 \mathrm{~s}$ depolarizing current steps and showed that NPY neurons had relatively linear input-output functions (Fig. 3B). NPY neurons had an average resting membrane potential of $-63.4 \pm 3.8 \mathrm{mV}$ (Fig. $3 \mathrm{C1}$ ) and exhibited relatively low expression of $I_{h}$ current, with voltage sag ratios (steady-state/ peak) of $0.89 \pm 0.09$ and $0.90 \pm 0.09$ measured from current steps that elicited peak hyperpolarization of $-91.1 \pm 1.8 \mathrm{mV}$ and $-111.0 \pm 0.7 \mathrm{mV}$, respectively (Fig. 3C2). In addition, NPY neurons exhibited moderate membrane time constants (15.9 \pm $8.6 \mathrm{~ms}$; Fig. 3C3) and input resistances $\left(R_{p k}=238.7 \pm 124.0 \mathrm{M} \Omega\right.$, $R_{s s}=249.5 \pm 167.0 \mathrm{M} \Omega$, Fig. $3 C 4$ ), and cells that did not spontaneously fire had low rheobase values (46.7 $\pm 25.1 \mathrm{pA}$; Fig. 3C5).

Neurons were classified as having a sustained firing pattern if their spike frequency adaptation (SFA) ratio (last interspike interval/first interspike interval) was $<2$ and as adapting if their SFA ratio was $>2$ (Peruzzi et al., 2000). We found that $92.5 \%$ of NPY neurons ( $n=135$ of 146$)$ had a sustained firing pattern in response to current steps that elicited $\sim 5$ spikes and $89.7 \%$ ( $n=96$ of 107) had a sustained firing pattern in response to current steps that elicited $\sim 10$ spikes (Fig. 3C6). Because neurons in the IC exhibit diverse firing patterns, the finding that nearly all NPY neurons had sustained firing patterns supports the hypothesis that NPY neurons constitute a distinct neuron class.

Interestingly, $71.9 \%$ of NPY neurons fired spontaneously at rest, with a median firing rate of $0.22 \mathrm{~Hz}$ ( $n=87$ of 121 neurons, 
range: $0.01-28.06 \mathrm{~Hz}$, mean $=2.70 \mathrm{~Hz}$; Fig. 3D1). To determine whether spontaneous firing was driven by spontaneous synaptic input, we applied $10 \mu \mathrm{m}$ NBQX and $50 \mu \mathrm{M}$ D-AP5 to block AMPA and NMDA receptors and found that spontaneous firing remained in 7 of 8 neurons (Fig. 3D2). Since the IC also receives cholinergic, dopaminergic, and serotonergic afferents (Paloff and Usunoff, 2000; Hurley et al., 2002; Tong et al., 2005; Motts and Schofield, 2009), we cannot exclude the possibility that part or all of the remaining spontaneous firing was driven by nonglutamatergic inputs. However, the cell bodies of nonglutamatergic excitatory inputs were not present in our IC slices, meaning that acetylcholine, dopamine, or serotonin would be released through miniature synaptic events that would presumably produce small postsynaptic responses. Consistent with this, in the presence of NBQX and D-AP5, action potentials appeared to rise directly from the baseline, independent from EPSPs (Fig. 3D3). While we will investigate the precise mechanisms underlying NPY neuron spontaneous firing in a future study, the present results show that most NPY neurons have a strong tendency to fire in the absence of glutamatergic inputs, suggesting that NPY neurons provide a tonic level of GABAergic and NPY signaling in the IC.

\section{NPY neurons have stellate morphology}

Using biocytin-streptavidin staining, we reconstructed the morphology of 49 NPY neurons. Twenty-four of these neurons were located in the ICc, 18 in ICd, and 7 in IClc. Figure 4 shows examples of the morphology of NPY neurons in the ICc, ICd, and IClc. For comparison purposes, neurons are oriented as they would appear in a coronal section of the left IC viewed from a caudal perspective. Visual inspection suggested that NPY neurons had a stellate morphology in all subdivisions. This is expected for ICd and IClc, where most neurons are stellate, but in the ICc, only $15 \%-20 \%$ of neurons have stellate morphology (Oliver and Morest, 1984; Oliver et al., 1991).

We used several quantitative approaches to test whether the dendritic arbors of NPY neurons in the ICc were consistent with the stellate morphology predicted by visual inspection. First, we used $2 \mathrm{D}$ principal component analysis (PCA) to determine the orientation and extent of the "length" (first principal direction) and "width" (second principal direction) axes of each NPY neuron within the coronal plane (Fig. 5A). In the mouse, the isofrequency laminae extend at a $\sim 40^{\circ}-50^{\circ}$ angle relative to the horizontal plane, and it is expected that the long axis of diskshaped neurons extends parallel to the isofrequency laminae (Stiebler and Ehret, 1985). Our data show that the long axis of
NPY neurons in the ICc did not have a preferred orientation, and only 3 of 24 neurons had a long axis oriented between $30^{\circ}$ and $60^{\circ}$ (Fig. $5 B$ ).

Second, because the dendrites of ICc stellate neurons typically extend across more than one isofrequency lamina, we measured how far ICc NPY neurons extended their dendrites perpendicular to a $45^{\circ}$ laminar plane. Although the width of isofrequency laminae has not been determined in mice, the distance between laminae in rats presumably provides a conservative estimate for mice and ranges between 90 and $150 \mu \mathrm{m}$ (Malmierca et al., 1993). We found that only 4 of 24 ICc NPY neurons had dendritic arbors that extended $<90 \mu \mathrm{m}$ perpendicular to the laminar plane, and only 9 of 24 had dendrites that extended $<150 \mu \mathrm{m}$ perpendicular to the laminar plane (Fig. $5 C$ ). This suggests that the majority of ICc NPY neurons have dendritic arbors that extend across two or more isofrequency laminae.

Third, we performed 3D PCA on the $x, y, z$ coordinate set for each ICc NPY neuron to determine the length (extent along the first principal direction) and width (extent along the second principal direction) of each neuron in three dimensions. We then calculated the length-to-width ratio of the dendritic arbor. 
A
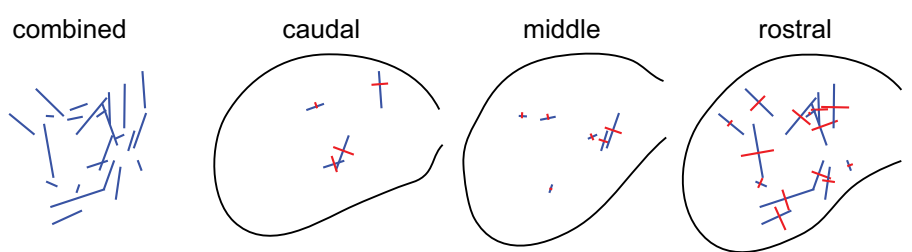

B

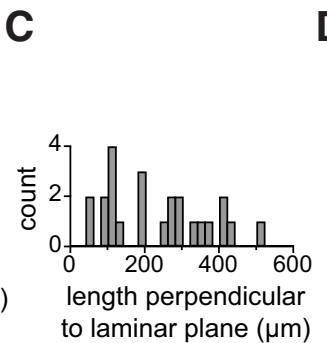

\section{D}

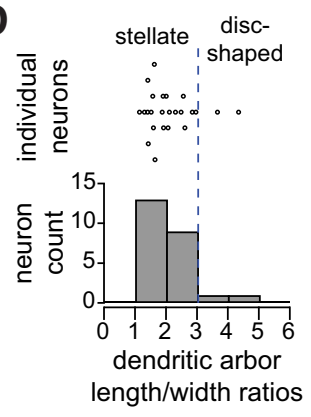

E
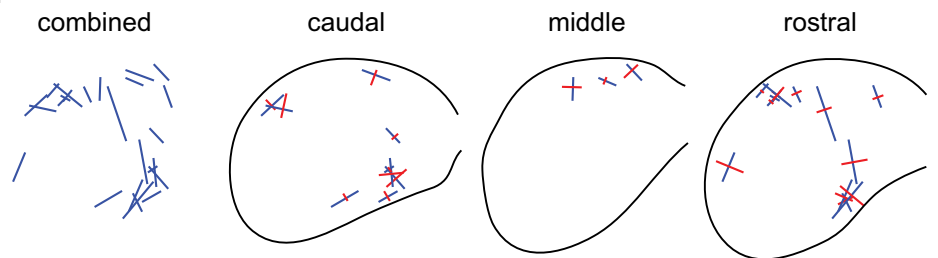

$\mathbf{F}$

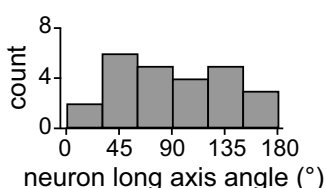

G

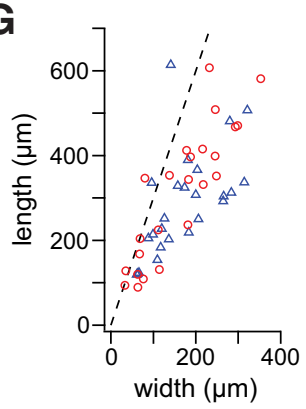

H

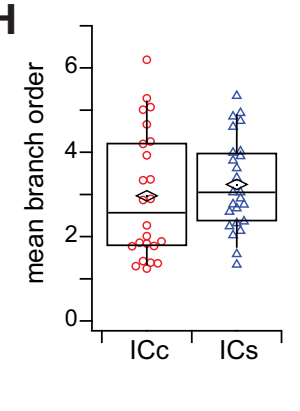

Figure 5. NPY neurons are a class of stellate cells. $A$, Orientation of the dendritic arbors of NPY neurons from the ICC. Combined: all reconstructed neurons from the ICC $(n=24)$. Blue lines indicate the orientation and length of the longest axis (first principal direction) of each neuron determined using 2D PCA. Caudal, middle, and rostral: orientation of dendritic fields separated according to position along the rostrocaudal axis of the ICC. Blue lines indicate the longest axis. Perpendicular red lines indicate the second longest axis (second principal direction) as determined by 2D PCA. B, Angular orientation of the long axis for every reconstructed NPY neuron located in the ICc. Angles indicate counterclockwise rotation relative to the medial-lateral (horizontal) axis. C, Extent of dendritic arbors of ICC NPY neurons perpendicular to the plane of the isofrequency laminae $\left(45^{\circ}\right) . D, 3 D$ PCA was used to determine the length and width axes of NPY neurons in the ICC; $92 \%$ (22 of 24) of NPY neurons had a length-to-width ratio $<3$, indicating that they are stellate cells. $\boldsymbol{E}$, Orientation of the dendritic fields of NPY neurons from the ICd and IClc (combined as ICs). Colors of lines and arrangement of sections are the same as in $\boldsymbol{A}$. $\boldsymbol{F}$, Angular orientation of the long axis for every reconstructed NPY neuron located in the ICS $(n=25)$. Angles indicate counterclockwise rotation relative to the medial-lateral (horizontal) axis. $\mathbf{G}$, Comparison of the length/width ratio between NPY neurons in the ICC (red) and ICs (blue). Dashed line indicates length/width ratio $=3$. $\boldsymbol{H}$, Comparison of the mean dendritic branch order between NPY neurons in the ICC and ICs.

Oliver et al. (1991) showed that stellate neurons have a lengthto-width ratio $<3$ and disk-shaped neurons have a ratio $\geq 3$. We found that 22 of 24 NPY neurons (92\%) in the ICc had a lengthto-width ratio $<3$, consistent with the hypothesis that NPY neurons are stellate (Fig. 5D). Combined, the above quantitative analyses support the conclusion that ICc NPY neurons are a class of stellate cells. Because stellate cells represent $15 \%-20 \%$ of ICc neurons (Oliver and Morest, 1984; Oliver et al., 1991) and our stereology data (Fig. 1G-I) indicate that NPY neurons represent
$7.6 \%$ of neurons in the ICc, NPY neurons represent $38 \%-50 \%$ of stellate neurons in the ICc.

We also performed 2D PCA on NPY neurons located in the ICd and IClc (combined as ICs; Fig. 5E). As in the ICc, the longest axis of NPY neurons in the ICs did not exhibit a preferred orientation (Fig. 5F). Instead, the long axes of ICs NPY neurons appeared to run parallel to the outer edge of the IC. Next, we compared the morphology of NPY neurons in the ICc versus those in the ICs. Interestingly, no difference was observed in the dendritic length/width ratio (ICc: $2.10 \pm 0.78$; ICs: $1.84 \pm 0.74$; twotailed Mann-Whitney-Wilcoxon test, $p=$ 0.160 ; Fig. $5 G$ ) or the mean branch order number (ICc: $2.97 \pm 1.52$; ICs: $3.24 \pm 1.10$; two-tailed Mann-Whitney-Wilcoxon test, $p=0.287$; Fig. $5 H$ ). Thus, there were no gross morphologic differences between NPY neurons in the ICc and ICs.

\section{NPY neurons project to the MG}

Most IC neurons, including GABAergic IC neurons, are believed to project to targets outside the IC (Oliver et al., 1991). As a population, GABAergic IC cells project to multiple extrinsic targets, including the MG, the superior colliculus, and the contralateral IC (for review, see Schofield and Beebe, 2019). In order to investigate whether NPY neurons project within the IC-MG pathway, the main ascending pathway for auditory perception, we placed red RBs into the MG in NPY-hrGFP mice. Figure $6 \mathrm{~A}$ shows an example in which the injection was centered on the dorsal MG. Across 7 animals, the injections included all major MG subdivisions. In every case, RB-labeled cells were numerous in the IC and included cells that were double-labeled with $\mathrm{RB}$ and GFP, identifying NPY cells that project to the MG (Fig. 6B,C, white arrows). Such double-labeled cells were present in all subdivisions of the ipsilateral IC. We also observed RBlabeled cells that were not $\mathrm{NPY}^{+}$(Fig. 6B,C, magenta arrows), indicating that there are many non-NPY ${ }^{+}$cells within the IC-ipsilateral MG pathway, consistent with previous reports that non-GABAergic (i.e., glutamatergic) IC cells also project to the MG. Finally, we observed NPY cells that did not contain RB (Fig. 6B, green arrows); these neurons could project to the MG but were not labeled by the MG injection, or they could represent IC neurons that project to a target other than the MG.

\section{$\mathrm{Y}_{1} \mathrm{R}$ neurons are glutamatergic neurons with heterogeneous} intrinsic physiology

Previous studies suggest that $\mathrm{Y}_{1}$ Rs are expressed in the IC (Kishi et al., 2005; Eva et al., 2006), but the neurotransmitter content and intrinsic physiology of the neurons expressing $\mathrm{Y}_{1} \mathrm{Rs}$ are 


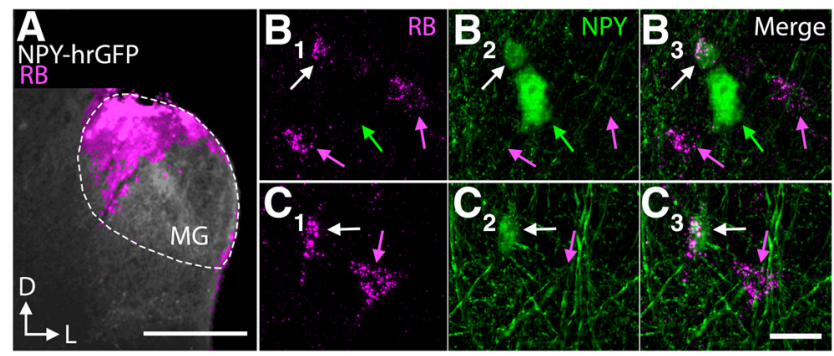

Figure 6. NPY neurons participate in the IC-MG pathway. $\boldsymbol{A}$, Low-magnification micrograph showing an injection of RBs (magenta) into the MG of an NPY-hrGFP mouse. To aid in the visualization of the RB injection, hrGFP is shown in white. White dotted outline indicates the approximate bounds of the MG. Top, Dorsal. Right, Lateral. Scale bar, $500 \mu \mathrm{m}$. B, C, High-magnification photomicrographs showing double-labeled (RB-labeled/NPY ${ }^{+}$) neurons (white arrows) in the IC ipsilateral to the injected MG. Nearby neurons could be single-labeled for RB (magenta arrows; $\boldsymbol{B}_{1}, \boldsymbol{C}_{1}$ ) or GFP (green arrow; $\boldsymbol{B}_{2}$ ). $\boldsymbol{B}_{1}, \boldsymbol{C}_{1}$ show RB labeling. $\boldsymbol{B}_{2}, \boldsymbol{C}_{\mathbf{2}}$ show GFP fluorescence. $\boldsymbol{B}_{3}, \boldsymbol{C}_{\mathbf{3}}$ show merged images. Scale bar, $20 \mu \mathrm{m}$.

unknown. We used Npy1r ${ }^{\text {cre }} \mathrm{x}$ Ai14 mice to label $\mathrm{Y}_{1} \mathrm{R}$-expressing neurons with the fluorescent protein tdTomato (Padilla et al., 2016) and found that $Y_{1} R$ neurons are broadly distributed in the IC (Fig. $7 A, B$ ). To investigate the neurotransmitter content of $\mathrm{Y}_{1} \mathrm{R}$ neurons, we performed immunofluorescence with antiGAD67 on brain slices from two Npy1 ${ }^{\text {cre }} \mathrm{x}$ Ai14 mice, aged P58. Using design-based stereology, we found that, across 2148 tdTomato $^{+}$neurons, only 24 neurons colabeled for GAD67 (1.1\%; Table 4), suggesting that all, or nearly all, $\mathrm{Y}_{1} \mathrm{R}$ neurons in the IC are glutamatergic (Fig. $7 C-E$ ). Interestingly, we also observed that blood vessels in the IC were lined with non-neuronal cells expressing $\mathrm{Y}_{1} \mathrm{R}$ (Fig. $7 A-E$, blue arrowheads). Because NPY induces vasoconstriction in other brain regions (Bao et al., 1997; Cauli et al., 2004), NPY signaling might regulate blood flow in the IC, an important hypothesis we will pursue in a future study.

We next targeted whole-cell current-clamp recordings to $\mathrm{Y}_{1} \mathrm{R}$ expressing neurons in acute brain slices from $N p y 1 r^{\text {cre }}$ x Ail4 mice. In contrast to the rather homogeneous intrinsic properties of NPY-hrGFP neurons, we found that $\mathrm{Y}_{1} \mathrm{R}$ neurons exhibited two distinct firing patterns that correlated with distinct intrinsic physiological properties. Slightly more than half of $\mathrm{Y}_{1} \mathrm{R}$ neurons exhibited a sustained firing pattern in response to depolarizing current steps, whereas the remaining $\mathrm{Y}_{1} \mathrm{R}$ neurons exhibited an adapting firing pattern (34 of 50 neurons had a SFA ratio $<2$, and 16 of 50 neurons had an SFA ratio $>2$; Fig. 7F,G1; SFA ratio calculated with $\sim 5$ spikes comparing sustained versus adapting neurons, two-tailed $t$ test, $p=0.0002$; SFA ratio calculated with $\sim 10$ spikes, two-tailed $t$ test, $p=0.002$; Bonferroni-corrected $\alpha=$ $0.00625)$. Sustained $Y_{1} R$ neurons had an average resting membrane potential of $-69.0 \pm 6.6 \mathrm{mV}$, whereas adapting $\mathrm{Y}_{1} \mathrm{R}$ neurons trended toward a more depolarized resting membrane potential of $-65.8 \pm 6.1 \mathrm{mV}$ (Fig. 7G2; two-tailed $t$ test, $p=0.104$ Bonferroni-corrected $\alpha=0.00625)$. Adapting $\mathrm{Y}_{1} \mathrm{R}$ neurons had a more prominent voltage sag in response to hyperpolarizing current steps compared with sustained neurons (Fig. $7 F$, right), with voltage sag ratios (steady-state/peak) of $0.89 \pm 0.1$ for sustained neurons and $0.63 \pm 0.2$ for adapting neurons (two-tailed $t$ test, $p=0.0004$; Bonferroni-corrected $\alpha=0.00625$ ). These values were measured from current steps that elicited peak hyperpolarization of $-91.0 \pm 1.3 \mathrm{mV}$ and $-91.0 \pm 0.4 \mathrm{mV}$, respectively (Fig. 7G3). In addition, sustained $\mathrm{Y}_{1} \mathrm{R}$ neurons exhibited a moderate membrane time constant $(17.0 \pm 8.8 \mathrm{~ms})$, while adapting neurons had a significantly faster membrane time constant $(5.8 \pm 4.3 \mathrm{~ms}$, Fig. 7G4; two-tailed $t$ test, $p=2 \times 10^{-6}$; Bonferroni-corrected $\alpha=$ $0.00625)$. Sustained neurons had significantly higher input resistances than adapting neurons (Fig. 7G5; sustained neurons: $R_{p k}=275.1 \pm 109.8 \mathrm{M} \Omega, R_{s s}=270.2 \pm 140.1 \mathrm{M} \Omega$; adapting neurons: $R_{p k}=134.3 \pm 60.0 \mathrm{M} \Omega, R_{s s}=95.1 \pm 67.3 \mathrm{M} \Omega ; R_{p k}$, twotailed $t$ test, $p=4 \times 10^{-7} ; R_{s S}$, two-tailed $t$ test, $p=7 \times 10^{-7}$; Bonferroni-corrected $\alpha=0.00625)$. $\mathrm{Y}_{1} \mathrm{R}$ neurons rarely spontaneously fired at rest and had moderate rheobase values that were higher for adapting neurons compared with sustained neurons $(59.4 \pm 46.6 \mathrm{pA}$ for sustained neurons and $105.7 \pm 53.3 \mathrm{pA}$ for adapting neurons, Fig. 7G6; two-tailed $t$ test, $p=0.00621$; Bonferroni-corrected $\alpha=0.00625)$. Based on the numerous significant differences between adapting and sustained $Y_{1} R$ neurons, we propose that $Y_{1}$ Rs are expressed by at least two distinct types of glutamatergic neurons in the IC.

\section{NPY hyperpolarizes a subset of $Y_{1} R$-expressing neurons in the IC}

NPY signaling is an important modulator of neuronal excitability in several brain regions (Colmers and Bleakman, 1994; Chee et al., 2010; Chen et al., 2019). We therefore hypothesized that NPY signaling alters the excitability of $\mathrm{Y}_{1} \mathrm{R}$ neurons in the IC. To test this hypothesis, we targeted current-clamp recordings to $Y_{1} R$ neurons while including $1 \mu \mathrm{M}$ TTX in the bath to block endogenous release of NPY from spontaneously active NPY neurons. We found that bath application of $\left[\mathrm{Leu}^{31}, \mathrm{Pro}^{34}\right]-\mathrm{NPY}$, a high-affinity $\mathrm{Y}_{1} \mathrm{R}$ agonist, hyperpolarized $V_{\text {rest }}$ in $50 \%$ of $\mathrm{Y}_{1} \mathrm{R}$ neurons by an average of $-6.2 \pm 4.3 \mathrm{mV}(n=5$ of 10 neurons; two-tailed paired $t$ test comparing baseline $V_{\text {rest }}$ with treatment $V_{\text {rest }}$, $p=0.032$; Fig. $8 A$ ). A neuron was considered sensitive to [Leu ${ }^{31}$, Pro $\left.{ }^{34}\right]$-NPY when $V_{\text {rest }}$ hyperpolarized $>2 \mathrm{mV}$ below baseline in the presence of the drug. Neurons that did not respond to the drug exhibited a $-0.5 \pm 0.8 \mathrm{mV}$ change in $V_{\text {rest }}$, which was not significantly different from the baseline $V_{\text {rest }}(n=5$ of 10 neurons; two-tailed paired $t$ test, $p=0.248$ ). In control experiments performed with a vehicle solution, there was no significant change in $V_{\text {rest }}$ between the baseline and vehicle periods $(0.2 \pm 1.3 \mathrm{mV}, n=5$; two-tailed paired $t$ test, $p=0.694$; Fig. $8 B)$. Neurons that responded to $\left[\mathrm{Leu}^{31}, \mathrm{Pro}^{34}\right]$-NPY exhibited a significantly different change in $V_{\text {rest }}$ compared with neurons treated with vehicle (two-tailed Mann-Whitney-Wilcoxon test, $p=0.007$; Fig. $8 C$ ). After a 30-50 min washout period, the $V_{\text {rest }}$ of all cells that responded to $\left[\mathrm{Leu}^{31}\right.$, Pro $\left.{ }^{34}\right]$-NPY returned to baseline ( $n=5$, two-tailed paired $t$ test, $p=0.333$; data not shown).

We next tested whether faster and more local delivery of $\left[\mathrm{Leu}^{31}, \mathrm{Pro}^{34}\right]$-NPY via a puffer pipette would lead to more consistent effects on the $V_{\text {rest }}$ of $\mathrm{Y}_{1} \mathrm{R}$ neurons. Indeed, we found that puff application of [Leu ${ }^{31}$, Pro $\left.{ }^{34}\right]$-NPY hyperpolarized the $V_{\text {rest }}$ of $89 \%$ of $\mathrm{Y}_{1} \mathrm{R}$ neurons by an average of $-3.1 \pm 2.0 \mathrm{mV}(n=8$ of 9 neurons; two-tailed paired $t$ test comparing baseline $V_{\text {rest }}$ with treatment $V_{\text {rest }}, p=0.007$; Fig. $8 D$ ). In control experiments, there was no significant difference between baseline $V_{\text {rest }}$ and $V_{\text {rest }}$ during vehicle application $\left(n=7\right.$; mean change in $V_{\text {rest }}=$ $0.3 \pm 1.2 \mathrm{mV}$; two-tailed paired $t$ test comparing baseline $V_{\text {rest }}$ with vehicle $V_{\text {rest }}, p=0.57$; Fig. $8 E$ ). As observed in the bath application experiment, there was a significantly different change in $V_{\text {rest }}$ between neurons treated with [ $\left.\mathrm{Leu}^{31}, \mathrm{Pro}^{34}\right]-\mathrm{NPY}$ and neurons treated with vehicle (two-tailed Mann-WhitneyWilcoxon test, $p=0.0006$; Fig. $8 F$ ).

Together, these results show that NPY signaling, by hyperpolarizing the resting membrane potential of a population $\mathrm{Y}_{1} \mathrm{R}$ expressing glutamatergic neurons, provides a novel mechanism 

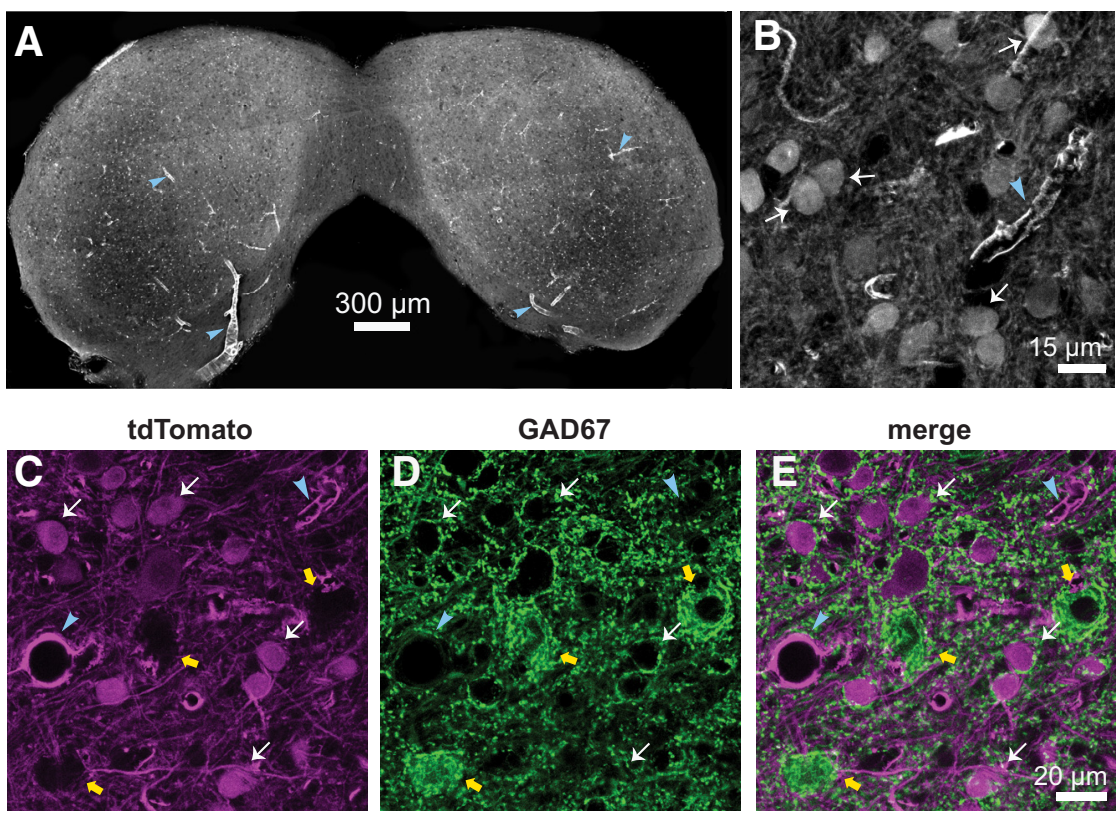

$\mathbf{F}$
Sustained
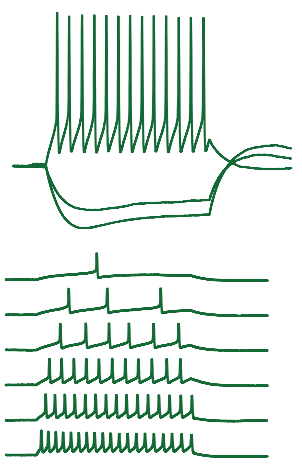

Adapting
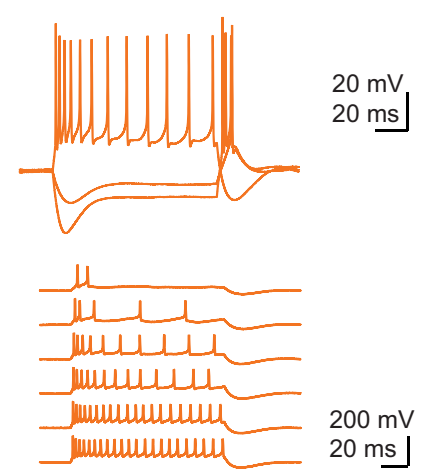

G

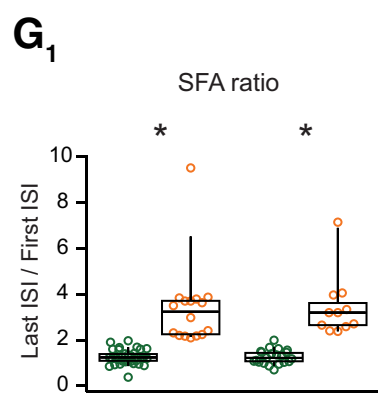

$\mathbf{G}_{2}$

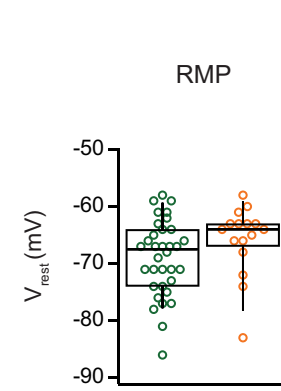

\section{$\mathbf{G}_{3}$}

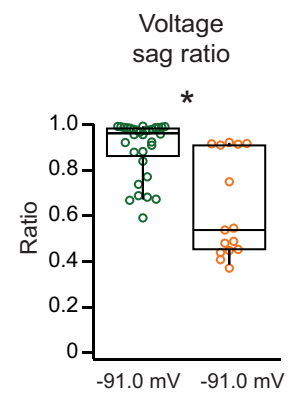

$\mathbf{G}_{4}$

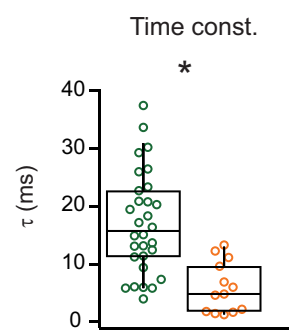

$\mathbf{G}_{5}$

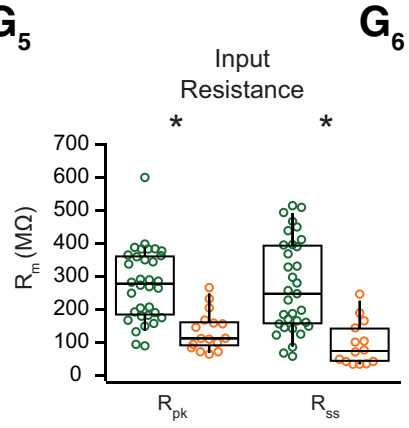

to dampen excitability in the IC. Since NPY neurons express NPY (Fig. $1 A-C$ ), it is likely that at least some NPY signaling in the IC originates from local NPY neurons.

\section{Discussion}

We identified NPY neurons as a novel class of inhibitory principal neurons in the IC. NPY neurons are GABAergic, produce NPY, are present throughout the IC, and have stellate morphology with dendrites that cross isofrequency laminae in the ICc. NPY neurons account for approximately one-third of GABAergic neurons in the IC and $38 \%-50 \%$ of stellate neurons in the ICc. NPY neurons fired spontaneously at rest, suggesting that they might tonically release GABA and NPY onto postsynaptic targets. Retrograde labeling showed that NPY neurons project to the ipsilateral MG. We also found the first evidence that NPY signaling can regulate excitability in the IC. We showed that $\mathrm{Y}_{1}$ Rs are expressed by glutamatergic neurons that are widely distributed throughout the IC and which likely consist of two or more neuron types. Intriguingly, most $\mathrm{Y}_{1} \mathrm{R}$ neurons were hyperpolarized by application of a high-affinity $\mathrm{Y}_{1} \mathrm{R}$ agonist, suggesting that NPY signaling in the IC might dampen activity through selective effects on an excitatory population of neurons. Thus, NPY neurons represent a major new class of IC inhibitory neurons that may use both GABAergic and NPY signaling to regulate activity levels in the IC and MG.

\section{NPY neurons are a distinct class of IC neurons}

Identification of fundamental neuron classes is essential for understanding the organization and function of neural

throughout the IC. $\boldsymbol{B}$, Higher-magnification image from the same section as in $\boldsymbol{A}$. White arrows indicate cell bodies of neurons that were tdTomato $^{+}$, indicating that they expressed $Y_{1} R$. Blue arrowheads indicate blood vessels lined with tdTomato ${ }^{+}$cells. $\boldsymbol{C}, \boldsymbol{D}, \boldsymbol{E}$, Confocal images of a coronal IC section from an Npy $7 r^{\text {re }}$ x Ai14 mouse. tdTomato ${ }^{+}$cells (C) did not colabel with GAD67 staining (D), suggesting that the $Y_{1} R$ neurons are glutamatergic $(\boldsymbol{E}$, merge). Yellow arrows indicate tdTomato ${ }^{-} / \mathrm{GAD} 7^{+}$neurons. $\boldsymbol{F}, \mathrm{Y}_{1} \mathrm{R}$ neurons exhibited one of two different firing patterns in response to depolarizing current steps: sustained (left, green) or adapting (right, orange). $G$, Sustained and adapting $Y_{1} R$ neurons exhibited heterogeneous intrinsic physiological properties. $\boldsymbol{G}_{1}$, SFA ratio. $\boldsymbol{G}_{2}$, Resting membrane potential. $\boldsymbol{G}_{3}$, Voltage sag ratio. $\boldsymbol{G}_{4}$, Membrane time constant. $\boldsymbol{G}_{5}$, Input resistance. $G_{6}$, Rheobase. Boxplots represent median, $25^{\text {th }}$ and $75^{\text {th }}$ percentile (box), and $9^{\text {th }}$ and $91^{\text {st }}$ percentile (whiskers). ${ }^{*} p<0.00625$.
Figure 7. $Y_{1} R$-expressing neurons are glutamatergic and exhibit sustained or adapting firing. $\boldsymbol{A}$, Tile scan image of tdTomato expression in a coronal section from an Npy1r $r^{\text {re }}$ x Ai14 mouse. tdTomato ${ }^{+}$neurons were broadly distributed 
circuits in the brain. Previous studies showed that the IC contains a large and diverse population of GABAergic neurons. These efforts relied on neuronal morphology (Oliver et al., 1994), in vivo physiology (Ono et al., 2017), neurochemical and extracellular markers (Ito et al., 2009; Beebe et al., 2016), or a combination of morphology, physiology, and neurotransmitter content (Ono et al., 2005). However, these studies yielded different results, and it is unclear whether the types of GABAergic neurons they defined represent functionally meaningful groups. Because the neuron groups identified in these studies lack molecular markers, they are not readily accessible for targeted manipulations with optogenetics and other molecular tools.

Combining molecular markers with physiological and anatomic parameters has become key to identifying functionally relevant classes of neurons (Tremblay et al., 2016; Zeng and Sanes, 2017). Through such an approach, we identified NPY neurons as a distinct subtype of GABAergic stellate neurons. NPY neurons are selectively labeled in NPY-hrGFP mice and share a common neurotransmitter content, neuropeptide expression, stellate morphology, and similar physiological features. Ninety-nine percent of NPY neurons immunostained for GAD67, and 95\% immunostained for NPY. Ninety-two percent of ICc NPY neurons met the criteria for stellate morphology. In our recordings, $90 \%-92 \%$ of NPY neurons had a sustained firing pattern. Previously, Ono et al. (2005) reported that $\mathrm{GAD}^{+} 7^{+}$neurons exhibited four firing patterns: regular sustained, buildup/ pauser, and transient with or without after-depolarizations. Our results show that NPY neurons fit into the regular sustained category, which included $43 \%$ of $\mathrm{GAD} 7^{+}$neurons in the Ono et al. (2005) study.

Most NPY neurons fired spontaneously at rest. Interestingly, spontaneous firing has been reported for NPY neurons in hypothalamus (van den Top et al., 2004), striatum (Partridge et al., 2009), and amygdala (Song et al., 2016). This suggests that NPY neurons in multiple brain regions might preferentially express a common suite of ion channels that promotes spontaneous firing. The spontaneous firing of IC NPY neurons indicates that NPY neurons in vivo might provide a constant tone of GABA and NPY to the IC and MG. This inhibitory tone might be rapidly upregulated or downregulated by inputs that modulate the activity levels of NPY neurons, providing a mechanism for NPY neurons to increase or decrease the overall excitability in postsynaptic brain regions.

\section{NPY neurons include GABAergic projection neurons}

In many brain regions, GABAergic neurons are interneurons (Tremblay et al., 2016), but in the IC, GABAergic cells contribute substantially to long-range projections to the MG and other targets (Winer et al., 1996; Peruzzi et al., 1997; St. Marie et al., 1997; Ito et al., 2009; Mellott et al., 2014, 2018; Beebe et al., 2018). The projection to MG is particularly interesting because other regions of primary sensory thalamus do not receive ascending inhibitory input (Halassa and Acsády, 2016). $\mathrm{Y}_{1} \mathrm{R}$ is expressed throughout all MG subdivisions (Kishi et al., 2005), and we observed tdTomato expression in a population of MG neurons in Npylr ${ }^{\text {cre }}$ x Ail4 mice (data not shown). Our retrograde experiments confirmed that NPY neurons project to the
Table 4. $Y_{1} R$ neurons are glutamatergic ${ }^{a}$

\begin{tabular}{lllccl}
\hline Animal & Slice $(n)$ & $\begin{array}{l}\text { No. of } \\
\text { tdTomato }^{+}\end{array}$ & $\begin{array}{l}\text { No. of } \\
\text { GAD67 }^{+}\end{array}$ & $\begin{array}{l}\text { No. of } \\
\text { colabel }\end{array}$ & $\begin{array}{l}\text { \% tdTomato } \\
\text { colabeled }\end{array}$ \\
\hline P58 male 1 & 1 (caudal) & 414 & 45 & 5 & 1.2 \\
& 2 (medial) & 221 & 32 & 1 & 0.5 \\
& 3 (rostral) & 212 & 28 & 3 & 1.4 \\
& Total & 847 & 105 & 9 & 1.1 \\
P58 male 2 & 1 (caudal) & 474 & 39 & 4 & 0.8 \\
& 2 (medial) & 472 & 41 & 6 & 1.3 \\
& 3 (rostral) & 355 & 52 & 5 & 1.4 \\
& Total & 1301 & 132 & 15 & 1.2 \\
Grand total & & 2148 & 237 & 24 & 1.1 \\
\hline
\end{tabular}

${ }^{a}$ Across 2 mice, an average of $1.1 \%$ of tdTomato ${ }^{+}$neurons were labeled with an antibody against GAD67.
A

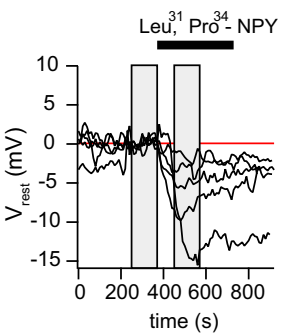

D

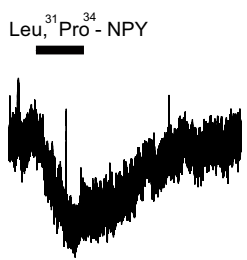

B

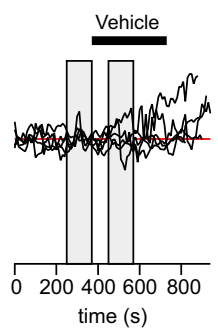

$\mathbf{E}$

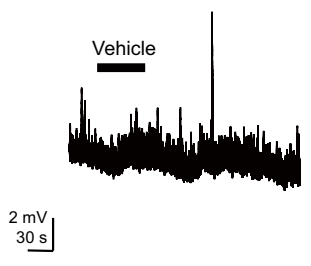

C

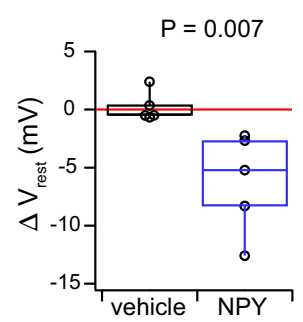

$\mathbf{F}$

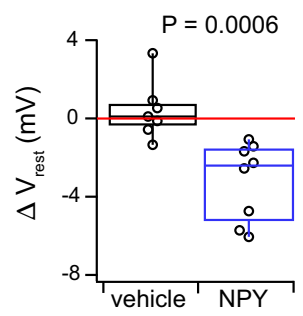

Figure 8. $\left[\right.$ Leu $\left.^{31}, \mathrm{Pro}^{34}\right]$-NPY hyperpolarized $V_{\text {rest }}$ in a subset of $\mathrm{Y}_{1} R$-expressing $\mathrm{IC}$ neurons. $\boldsymbol{A}, \boldsymbol{B}$, Graphs represent 15 min of recording from tdTomato ${ }^{+} Y_{1} R$ neurons. Gray bars represent periods in which baseline (left bar) and treatment (right bar) measurements were made. $A$, Bath application of $500 \mathrm{~nm}\left[\right.$ Leu $^{31}$, Pro $\left.{ }^{34}\right]-N P Y$ led to a hyperpolarizing comparing baseline $V_{\text {rest }}$ with treatment $\left.V_{\text {rest }} p=0.032\right)$. $\boldsymbol{B}$, Vehicle had no effect on the membrane potential of neurons $(0.2 \pm 1.3 \mathrm{mV}$ change; $n=5$; two-tailed paired $t$ test, $p=0.694)$. C, Boxplot represents the between vehicle- and [Leu ${ }^{31}$ Pro ${ }^{34}$ ]-NPY-treated neurons (two-tailed Mann-Whitney-Wilcoxon test, D. Puff application of [Leu ${ }^{31}, \mathrm{Pro}^{34}$-NPY led to a hyperpolarizing shift in the $V_{\text {res }}$ of $89 \%$ of tdTomato ment, $p=0.007)$. $E_{\text {Vehicle application }}$ had no effect on the membrane potential $(0.3+1.2 \mathrm{mV}$ rest with $n=7$ two-tailed paired $t$ test comparing baseline $V_{\text {rest }}$ to treatment, $\left.p=0.57\right)$. $\boldsymbol{F}$, Boxplots represent the change in $V_{\text {rest }}$ between vehicle and $\left[\mathrm{Leu}^{31}, \mathrm{Pro}^{34}\right]$-NPY-treated neurons (two-tailed Mann-Whitney-Wilcoxon test, $p=0.0006$ ). Boxplots represent median, 25th and 75th percentile (box), and 9th and 91st percentile (whiskers).

MG. GABAergic tectothalamic projections arise from multiple IC subdivisions and terminate in multiple MG subdivisions (Mellott et al., 2014), reflecting the parallel organization of the ascending system (Calford and Aitkin, 1983; Rouiller, 1997; Cai et al., 2019). As shown here, NPY has inhibitory effects that could complement GABAergic effects on postsynaptic targets. It will be interesting to determine whether the presence or absence of NPY corelease with GABA underlies some of the differences in collicular-driven inhibition of MG cells (Bartlett and Smith, 2002). Since NPY neurons are molecularly identifiable, it is now possible to use optogenetics and other targeted approaches to test how specific inhibitory projections from the IC shape activity in the MG and other targets. 


\section{NPY neurons likely use both GABA and NPY signaling to regulate activity in the IC and MG}

NPY is widely expressed in the mammalian brain and is a potent modulator of neuronal activity. Previous brainwide screening studies suggested that NPY and $Y_{1} R s$ are expressed in the IC (Widdowson, 1993; Kishi et al., 2005; Eva et al., 2006), but the experiments conducted here are the first to characterize IC neurons that synthesize NPY and to test the effects of NPY signaling on IC neurons. $\mathrm{Y}_{1} \mathrm{Rs}$ tend to inhibit neurons when activated (Giesbrecht et al., 2010). Consistent with this, we found that bath application of $\left[\right.$ Leu $\left.^{31}, \mathrm{Pro}^{34}\right]$-NPY hyperpolarized $V_{\text {rest }}$ in $50 \%$ of the tested $Y_{1} R$-expressing neurons, and puff application hyperpolarized $V_{\text {rest }}$ in $89 \%$ of the tested neurons. $\mathrm{Y}_{1} \mathrm{R}$ activation can induce opening of $G$ protein-coupled inwardly rectifying potassium $\left(\mathrm{G}_{\text {IRK }}\right)$ channels, suggesting that $\mathrm{G}_{\mathrm{IRK}}$ activation might mediate the effects we observed (Sun et al., 2001; Paredes et al., 2003; Fu et al., 2004; Sosulina et al., 2008), although other mechanisms are possible (Giesbrecht et al., 2010; Villarroel et al., 2018). The fact that not all IC $Y_{1} R$ neurons were sensitive to NPY might be due to transient developmental expression of Cre in some neurons. Alternatively, nonresponsive neurons might respond to NPY in ways that we did not test.

Although our neuromodulation experiments focused on $Y_{1} R$ expressing neurons, other subtypes of YRs might also support NPY signaling in the IC. In particular, $\mathrm{Y}_{5} \mathrm{Rs}$ may be expressed in the IC (Dumont et al., 1998b). In hypothalamus, activation of $Y_{5} R s$ hyperpolarizes GnRH neurons (Xu et al., 2009), suggesting that $\mathrm{Y}_{5} \mathrm{R}$ and $\mathrm{Y}_{1} \mathrm{R}$ signaling may play similar inhibitory roles in the IC. In other brain areas, $\mathrm{Y}_{2} \mathrm{Rs}$ are located on presynaptic terminals and their activation can decrease glutamatergic or GABAergic transmission (Colmers et al., 1991; Mackay et al., 2019), but the expression of $\mathrm{Y}_{2} \mathrm{Rs}$ is either low (Dumont et al., 1998a; Primus et al., 1998) or absent in the IC (Trivedi et al., 2001; Stanić et al., 2006).

Beyond influencing auditory computations, the inhibitory effects of NPY signaling might serve diverse roles in the IC. In the hippocampus and amygdala, NPY released from GABAergic NPY neurons acts as an endogenous anticonvulsant (Colmers and Bleakman, 1994; Colmers and El Bahh, 2003; Noe' et al., 2007). Interestingly, the IC is the main site of initiation of audiogenic seizures (Ross and Coleman, 2000; Faingold, 2002), so NPY/GABA release might protect against hyperexcitability in the IC. Consistent with this, acoustic trauma leads to an increase in the expression of BDNF in the IC (Meltser and Canlon, 2010); and in other brain regions, BDNF can enhance NPY expression (Nawa et al., 1994; Takei et al., 1996; Wirth et al., 2005).

The IC also plays an important role in auditory task-related unconditioned fear responses (Muthuraju et al., 2014; de Oliveira et al., 2014) and anxiety (Silveira et al., 1993). In the amygdala (Sajdyk et al., 2008), cortex (Vollmer et al., 2016), and hippocampus (Li et al., 2017), NPY exhibits anxiolytic properties and regulates behaviors associated with fear. Thus, NPY neurons in the IC might regulate how IC circuits process unconditioned fear responses and how they are affected by behavioral states, such as anxiety.

Interestingly, in the IC of Npylr ${ }^{c r e}$ x Ail4 mice, we found strong labeling of cells associated with blood vessels (Fig. 7A-E). Previous studies showed that NPY induces contraction in cortical microvessels by activating $\mathrm{Y}_{1} \mathrm{Rs}$ (Abounader et al., 1999; Cauli et al., 2004) and plays a similar role in the cochlea (Carlisle et al., 1990). Since the IC is reported to be the most metabolically active brain region and to have the highest rate of blood flow in the brain (Sokoloff et al., 1977; Gross et al., 1987; Zeller et al., 1997), IC NPY neurons might play a critical role regulating energy homeostasis in the IC.

\section{NPY and VIP neurons account for more than half of ICc stellate neurons}

ICc stellate neurons have long been a subject of speculation, but their functional roles are almost completely unknown due to lack of an approach to target or selectively manipulate them during in vivo recordings or behavioral testing. Here, we have identified a distinct GABAergic cell type labeled in the NPY-hrGFP mouse. In addition, we recently identified VIP neurons as a novel class of glutamatergic stellate cells in the IC, and VIP neurons can be selectively manipulated in VIP-IRES-Cre mice (Goyer et al., 2019). VIP and NPY neurons represent $3.5 \%$ and $7.6 \%$ of neurons in the ICc, respectively, and combined they account for $\sim 55 \%-74 \%$ of ICc stellate neurons. Thus, for the first time, we are positioned to determine the functional roles of excitatory and inhibitory stellate neurons that together represent the majority of ICc stellate neurons.

\section{References}

Abounader R, Elhusseiny A, Cohen Z, Olivier A, Stanimirovic D, Quirion R, Hamel E (1999) Expression of neuropeptide Y receptors mRNA and protein in human brain vessels and cerebromicrovascular cells in culture. J Cereb Blood Flow Metab 19:155-163.

Acuna-Goycolea C, van den Pol AN (2005) Peptide YY(3-36) inhibits both anorexigenic proopiomelanocortin and orexigenic neuropeptide $\mathrm{Y}$ neurons: implications for hypothalamic regulation of energy homeostasis. J Neurosci 25:10510-10519.

Adams JC (1979) Ascending projections to the inferior colliculus. J Comp Neurol 183:519-538.

Bacci A, Huguenard JR, Prince DA (2002) Differential modulation of synaptic transmission by neuropeptide $\mathrm{Y}$ in rat neocortical neurons. Proc Natl Acad Sci USA 99:17125-17130.

Bao L, Kopp J, Zhang X, Xu ZQ, Zhang LF, Wong H, Walsh J, Hökfelt T (1997) Localization of neuropeptide Y Y1 receptors in cerebral blood vessels. Proc Natl Acad Sci USA 94:12661-12666.

Bartlett EL, Smith PH (2002) Effects of paired-pulse and repetitive stimulation on neurons in the rat medial geniculate body. Neuroscience 113:957-974.

Beebe NL, Mellott JG, Schofield BR (2018) Inhibitory projections from the inferior colliculus to the medial geniculate body originate from four subtypes of GABAergic cells. eNeuro 5:ENEURO.0406-18.2018.

Beebe NL, Young JW, Mellott JG, Schofield BR (2016) Extracellular molecular markers and soma size of inhibitory neurons: evidence for four subtypes of GABAergic cells in the inferior colliculus. J Neurosci 36:3988-3999.

Buentello DC, Bishop DC, Oliver DL (2015) Differential distribution of GABA and glycine terminals in inferior colliculus of rat and mouse. J Comp Neurol 523:2683-2697.

Cai D, Yue Y, Su X, Liu M, Wang Y, You L, Xie F, Deng F, Chen F, Luo M, Yuan K (2019) Distinct Anatomical Connectivity Patterns Differentiate Subdivisions of the Nonlemniscal Auditory Thalamus in Mice. Cereb Cortex 29:2437-2454.

Calford MB, Aitkin LM (1983) Ascending projections to the medial geniculate body of the cat: evidence for multiple, parallel auditory pathways through thalamus. J Neurosci 3:2365-2380.

Cant NB, Benson CG (2006) Organization of the inferior colliculus of the gerbil (Meriones unguiculatus): differences in distribution of projections from the cochlear nuclei and the superior olivary complex. J Comp Neurol 495:511-528.

Cant NB, Benson CG (2007) Multiple topographically organized projections connect the central nucleus of the inferior colliculus to the ventral division of the medial geniculate nucleus in the gerbil, Meriones unguiculatus. J Comp Neurol 503:432-453.

Carlisle L, Aberdeen J, Forge A, Burnstock G (1990) Neural basis for regulation of cochlear blood flow: peptidergic and adrenergic innervation of the spiral modiolar artery of the guinea pig. Hear Res 43:107-113.

Cauli B, Tong XK, Rancillac A, Serluca N, Lambolez B, Rossier J, Hamel E (2004) Cortical GABA interneurons in neurovascular coupling: relays for subcortical vasoactive pathways. J Neurosci 24:8940-8949. 
Chambers AR, Resnik J, Yuan Y, Whitton JP, Edge AS, Liberman MC, Polley DB (2016) Central gain restores auditory processing following near-complete cochlear denervation. Neuron 89:867-879.

Chee MJ, Myers MG Jr, Price CJ, Colmers WF (2010) Neuropeptide Y suppresses anorexigenic output from the ventromedial nucleus of the hypothalamus. J Neurosci 30:3380-3390.

Chen Y, Essner RA, Kosar S, Miller OH, Lin YC, Mesgarzadeh S, Knight Z (2019) Sustained NPY signaling enables AgRP neurons to drive feeding. Elife 8:e46348.

Chronwall BM, DiMaggio DA, Massari VJ, Pickel VM, Ruggiero DA, O'Donohue TL (1985) The anatomy of neuropeptide-Y-containing neurons in rat brain. Neuroscience 15:1159-1181.

Colmers WF, Klapstein GJ, Fournier A, St-Pierre S, Treherne KA (1991) Presynaptic inhibition by neuropeptide $\mathrm{Y}$ in rat hippocampal slice in vitro is mediated by a Y2 receptor. Br J Pharmacol 102:41-44.

Colmers WF, Bleakman D (1994) Effects of neuropeptide Y on the electrical properties of neurons. Trends Neurosci 17:373-379.

Colmers WF, El Bahh B (2003) Neuropeptide Y and epilepsy. Epilepsy Curr 3:53-58.

Coote EJ, Rees A (2008) The distribution of nitric oxide synthase in the inferior colliculus of guinea pig. Neuroscience 154:218-225.

de Oliveira AR, Colombo AC, Muthuraju S, Almada RC, Brandão ML (2014) Dopamine D2-like receptors modulate unconditioned fear: role of the inferior colliculus. PLoS One 9:e104228.

Dumont Y, Jacques D, Bouchard P, Quirion R (1998a) Species differences in the expression and distribution of the neuropeptide Y Y1, Y2, Y4, and Y5 receptors in rodents, guinea pig, and primate brains. J Comp Neurol 402:372-384.

Dumont Y, Fournier A, Quirion R (1998b) Expression and characterization of the neuropeptide Y Y5 receptor subtype in the rat brain. J Neurosci 18:5565-5574.

Eva C, Serra M, Mele P, Panzica G, Oberto A (2006) Physiology and gene regulation of the brain NPY Y1 receptor. Front Neuroendocrinol 27:308-339.

Faingold CL (2002) Role of GABA abnormalities in the inferior colliculus pathophysiology: audiogenic seizures. Hear Res 168:223-237.

Faye-Lund H, Osen KK (1985) Anatomy of the inferior colliculus in rat. Anat Embryol 171:1-20.

Foster NL, Mellott JG, Schofield BR (2014) Perineuronal nets and GABAergic cells in the inferior colliculus of guinea pigs. Front Neuroanat 7:53.

Fu LY, Acuna-Goycolea C, van den Pol AN (2004) Neuropeptide Y inhibits hypocretin/orexin neurons by multiple presynaptic and postsynaptic mechanisms: tonic depression of the hypothalamic arousal system. J Neurosci 24:8741-8751.

Giesbrecht CJ, Mackay JP, Silveira HB, Urban JH, Colmers WF (2010) Countervailing modulation of Ih by neuropeptide $\mathrm{Y}$ and corticotrophinreleasing factor in basolateral amygdala as a possible mechanism for their effects on stress-related behaviors. J Neurosci 30:16970-16982.

Goyer D, Silveira MA, George AP, Beebe NL, Edelbrock RM, Malinski PT, Schofield BR, Roberts MT (2019) A novel class of inferior colliculus principal neurons labeled in vasoactive intestinal peptide-Cre mice. eLife 8:e43770.

Gross PM, Sposito NM, Pettersen SE, Panton DG, Fenstermacher JD (1987) Topography of capillary density, glucose metabolism, and microvascular function within the rat inferior colliculus. J Cereb Blood Flow Metab 7:154-160.

Halassa MM, Acsády L (2016) Thalamic inhibition: diverse sources, diverse scales. Trends Neurosci 39:680-693.

Henry FE, Sugino K, Tozer A, Branco T, Sternson SM (2015) Cell type-specific transcriptomics of hypothalamic energy-sensing neuron responses to weight-loss. eLife 4:e09800.

Hurley LM, Thompson AM, Pollak GD (2002) Serotonin in the inferior colliculus. Hear Res 168:1-11

Ito T, Bishop DC, Oliver DL (2009) Two classes of GABAergic neurons in the inferior colliculus. J Neurosci 29:13860-13869.

Jinno S, Kosaka T (2003) Patterns of expression of neuropeptides in GABAergic nonprincipal neurons in the mouse hippocampus: quantitative analysis with optical dissector. J Comp Neurol 461:333-349.

Karagiannis A, Gallopin T, Dávid C, Battaglia D, Geoffroy H, Rossier J, Hillman EM, Staiger JF, Cauli B (2009) Classification of NPY-expressing neocortical interneurons. J Neurosci 29:3642-3659.
Keesom SM, Morningstar MD, Sandlain R, Wise BM, Hurley LM (2018) Social isolation reduces serotonergic fiber density in the inferior colliculus of female, but not male, mice. Brain Res 1694:94-103.

Kishi T, Aschkenasi CJ, Choi BJ, Lopez ME, Lee CE, Liu H, Hollenberg AN, Friedman JM, Elmquist JK (2005) Neuropeptide Y Y1 receptor mRNA in rodent brain: distribution and colocalization with melanocortin-4 receptor. J Comp Neurol 482:217-243.

Ledoux VA, Smejkalova T, May RM, Cooke BM, Woolley CS (2009) Estradiol facilitates the release of NPY to suppress hippocampus-dependent seizures. J Neurosci 29:1457-1468.

Li Q, Bartley AF, Dobrunz LE (2017) Endogenously released neuropeptide Y suppresses hippocampal short-term facilitation and is impaired by stressinduced anxiety. J Neurosci 37:23-37.

Mackay JP, Bompolaki M, DeJoseph MR, Michaelson SD, Urban JH, Colmers WF (2019) NPY2 receptors reduce tonic action potential-independent GABAB currents in the basolateral amygdala. J Neurosci 39:4909-4930

Malmierca MS, Blackstad TW, Osen KK, Karagülle T, Molowny RL (1993) The central nucleus of the inferior colliculus in rat: a Golgi and computer reconstruction study of neuronal and laminar structure. J Comp Neurol 333:1-27.

Marshall CJ, Desroziers E, McLennan T, Campbell RE (2017) Defining subpopulations of arcuate nucleus GABA neurons in male, female, and prenatally androgenized female mice. Neuroendocrinology 105:157-169.

Meininger V, Pol D, Derer P (1986) The inferior colliculus of the mouse: a Nissl and Golgi study. Neuroscience 17:1159-1179.

Mellott JG, Foster NL, Nakamoto KT, Motts SD, Schofield BR (2014) Distribution of GABAergic cells in the inferior colliculus that project to the thalamus. Front Neuroanat 7:53.

Mellott JG, Beebe NL, Schofield BR (2018) GABAergic and non-GABAergic projections to the superior colliculus from the auditory brainstem. Brain Struct Funct 223:1923-1936.

Meltser I, Canlon B (2010) The expression of mitogen-activated protein kinases and brain-derived neurotrophic factor in inferior colliculi after acoustic trauma. Neurobiol Dis 40:325-330.

Merchán M, Aguilar LA, Lopez-Poveda EA, Malmierca MS (2005) The inferior colliculus of the rat: quantitative immunocytochemical study of GABA and glycine. Neuroscience 136:907-925.

Milstein AD, Bloss EB, Apostolides PF, Vaidya SP, Dilly GA, Zemelman BV, Magee JC (2015) Inhibitory gating of input comparison in the CA1 microcircuit. Neuron 87:1274-1289.

Morest DK, Oliver DL (1984) The neuronal architecture of the inferior colliculus in the cat: defining the functional anatomy of the auditory midbrain. J Comp Neurol 222:209-236.

Motts SD, Schofield BR (2009) Sources of cholinergic input to the inferior colliculus. Neuroscience 160:103-114.

Muthuraju S, Nobre MJ, Saito VM, Brandao ML (2014) Distinct effects of haloperidol in the mediation of conditioned fear in the mesolimbic system and processing of unconditioned aversive information in the inferior colliculus. Neuroscience 261:195-206.

Nakagawa H, Ikeda M, Houtani T, Ueyama T, Baba K, Kondoh A, Yamamoto T, Yamashita T, Sugimoto T (1995) Immunohistochemical evidence for enkephalin and neuropeptide $\mathrm{Y}$ in rat inferior colliculus neurons that provide ascending or commissural fibers. Brain Res 690:236-240

Nawa H, Pelleymounter MA, Carnahan J (1994) Intraventricular administration of BDNF increases neuropeptide expression in newborn rat brain. J Neurosci 14:3751-3765.

Noben-Trauth K, Zheng QY, Johnson KR (2003) Association of cadherin 23 with polygenic inheritance and genetic modification of sensorineural hearing loss. Nat Genet 35:21-23.

Noe’ F, Nissinen J, Pitkänen A, Gobbi M, Sperk G, During M, Vezzani A (2007) Gene therapy in epilepsy: the focus on NPY. Peptides 28:377-383.

O’Donohue TL, Chronwall BM, Pruss RM, Mezey E, Kiss JZ, Eiden LE, Massari VJ, Tessel RE, Pickel VM, DiMaggio DA, Hotchkiss AJ, Crowley WR, Zukowska-Grojec Z (1985) Neuropeptide Y and peptide YY neuronal and endocrine systems. Peptides 6:755-768.

Oliver DL, Morest DK (1984) The central nucleus of the inferior colliculus in the cat. J Comp Neurol 222:237-264.

Oliver DL, Kuwada S, Yin TC, Haberly LB, Henkel CK (1991) Dendritic and axonal morphology of HRP-injected neurons in the inferior colliculus of the cat. J Comp Neurol 303:75-100. 
Oliver DL, Winer JA, Beckius GE, Saint Marie RL (1994) Morphology of GABAergic neurons in the inferior colliculus of the cat. J Comp Neurol 340:27-42.

Ono M, Yanagawa Y, Koyano K (2005) GABAergic neurons in inferior colliculus of the GAD67-GFP knock-in mouse: electrophysiological and morphological properties. Neurosci Res 51:475-492.

Ono M, Bishop DC, Oliver DL (2017) Identified GABAergic and glutamatergic neurons in the mouse inferior colliculus share similar response properties. J Neurosci 37:8952-8964.

Padilla SL, Qiu J, Soden ME, Sanz E, Nestor CC, Barker FD, Quintana A, Zweifel LS, Rønnekleiv OK, Kelly MJ, Palmiter RD (2016) Agouti-related peptide neural circuits mediate adaptive behaviors in the starved state. Nat Neurosci 19:734-741.

Palmer AR, Shackleton TM, Sumner CJ, Zobay O, Rees A (2013) Classification of frequency response areas in the inferior colliculus reveals continua not discrete classes. J Physiol 591:4003-4025.

Paloff AM, Usunoff KG (2000) Tyrosine hydroxylase-like immunoreactive synaptic boutons in the inferior colliculus of the cat. Ann Anat 182:423-426.

Paredes MF, Greenwood J, Baraban SC (2003) Neuropeptide Y modulates a $G$ protein-coupled inwardly rectifying potassium current in the mouse hippocampus. Neurosci Lett 340:9-12.

Partridge JG, Janssen MJ, Chou DY, Abe K, Zukowska Z, Vicini S (2009) Excitatory and inhibitory synapses in neuropeptide $\mathrm{Y}$-expressing striatal interneurons. J Neurophysiol 102:3038-3045.

Peruzzi D, Bartlett E, Smith PH, Oliver DL (1997) A monosynaptic GABAergic input from the inferior colliculus to the medial geniculate body in rat. J Neurosci 17:3766-3777.

Peruzzi D, Sivaramakrishnan S, Oliver DL (2000) Identification of cell types in brain slices of the inferior colliculus. Neuroscience 101:403-416.

Primus RJ, Yevich E, Gallager DW (1998) In vitro autoradiography of GTPg Sx binding at activated NPY receptors subtypes in the brain. Mol Brain Res 58:74-82.

Polgár E, Sardella TC, Watanabe M, Todd AJ (2011) Quantitative study of NPY-expressing GABAergic neurons and axons in rat spinal dorsal horn. J Comp Neurol 519:1007-1023.

Roa J, Herbison AE (2012) Direct regulation of GnRH neuron excitability by arcuate nucleus POMC and NPY neuron neuropeptides in female mice. Endocrinology 153:5587-5599.

Rose JB, Crews L, Rockenstein E, Adame A, Mante M, Hersh LB, Gage FH, Spencer B, Potkar R, Marr RA, Masliah E (2009) Neuropeptide Y fragments derived from neprilysin processing are neuroprotective in a transgenic model of Alzheimer's disease. J Neurosci 29:1115-1125.

Ross KC, Coleman JR (2000) Developmental and genetic audiogenic seizure models: behavior and biological substrates. Neurosci Biobehav Rev 24:639-653.

Rouiller EM (1997) Functional organization of the auditory pathways. The Central Auditory System (Ehret G, Romand R eds), pp 3-96. New York: Oxford University Press.

Rowan S, Todd AJ, Spike RC (1993) Evidence that neuropeptide Y is present in GABAergic neurons in the superficial dorsal horn of the rat spinal cord. Neuroscience 53:537-545.

Saint Marie RL, Stanforth DA, Jubelier EM (1997) Substrate for rapid feedforward inhibition of the auditory forebrain. Brain Res 765:173-176.

Sajdyk TJ, Johnson PL, Leitermann RJ, Fitz SD, Dietrich A, Morin M, Gehlert DR, Urban JH, Shekhar A (2008) Neuropeptide Y in the amygdala induces long-term resilience to stress-induced reductions in social responses but not hypothalamic-adrenal-pituitary axis activity or hyperthermia. J Neurosci 28:893-903.

Schofield BR, Beebe NL (2019) Subtypes of GABAergic cells in the inferior colliculus. Hear Res 376:1-10.

Silveira MC, Sandner G, Graeff FG (1993) Induction of Fos immunoreactivity in the brain by exposure to the elevated plus-maze. Behav Brain Res 56:115-118.

Sokoloff L, Reivich M, Kennedy C, Rosiers MH, Patlak CS, Pettigrew KD, Sakurada O, Shinohara M (1977) The $\left[{ }^{14} \mathrm{C}\right]$ deoxyglucose method for the measurement of local cerebral glucose utilization: theory, procedure, and normal values in the conscious and anesthetized albino rat1. J Neurochem 28:897-916.

Song SC, Beatty JA, Wilson CJ (2016) The ionic mechanism of membrane potential oscillations and membrane resonance in striatal LTS interneurons. J Neurophysiol 116:1752-1764.
Sosulina L, Schwesig G, Seifert G, Pape HC (2008) Neuropeptide Y activates a G-protein-coupled inwardly rectifying potassium current and dampens excitability in the lateral amygdala. Mol Cell Neurosci 39:491-498.

Stanić D, Brumovsky P, Fetissov S, Shuster S, Herzog H, Hökfelt T (2006) Characterization of neuropeptide Y2 receptor protein expression in the mouse brain: I. Distribution in cell bodies and nerve terminals. J Comp Neurol 499:357-390.

Stiebler I, Ehret G (1985) Inferior colliculus of the house mouse: I. A quantitative study of tonotopic organization, frequency representation, and tone-threshold distribution. J Comp Neurol 238:65-76.

Sturm JJ, Zhang-Hooks YX, Roos H, Nguyen T, Kandler K (2017) Noise trauma-induced behavioral gap detection deficits correlate with reorganization of excitatory and inhibitory local circuits in the inferior colliculus and are prevented by acoustic enrichment. J Neurosci 37:6314-6330.

Sun QQ, Huguenard JR, Prince DA (2001) Neuropeptide Y receptors differentially modulate G-protein-activated inwardly rectifying $\mathrm{K}^{+}$channels and high-voltage-activated $\mathrm{Ca}^{2+}$ channels in rat thalamic neurons. J Physiol 531:67-79.

Takei N, Sasaoka K, Higuchi H, Endo Y, Hatanaka H (1996) BDNF increases the expression of neuropeptide Y mRNA and promotes differentiation/ maturation of neuropeptide Y-positive cultured cortical neurons from embryonic and postnatal rats. Brain Res Mol Brain Res 37:283-289.

Tong L, Altschuler RA, Holt AG (2005) Tyrosine hydroxylase in rat auditory midbrain: distribution and changes following deafness. Hear Res 206:28-41.

Tong Q, Ye CP, Jones JE, Elmquist JK, Lowell BB (2008) Synaptic release of GABA by AgRP neurons is required for normal regulation of energy balance. Nat Neurosci 11:998-1000.

Trivedi PG, Yu H, Trumbauer M, Chen H, Van der Ploeg LH, Guan XM (2001) Differential regulation of neuropeptide $Y$ receptors in the brains of NPY knock-out mice. Peptides 22:395-403.

Tremblay R, Lee S, Rudy B (2016) GABAergic interneurons in the neocortex: from cellular properties to circuits. Neuron 91:260-292.

van den Pol AN, Yao Y, Fu LY, Foo K, Huang H, Coppari R, Lowell B, Broberger C (2009) Neuromedin B and gastrin releasing peptide excite arcuate nucleus neuropeptide $\mathrm{Y}$ neurons in a novel transgenic mouse expressing strong Renilla GFP in NPY neurons. J Neurosci 29:4622-4639.

van den Top T, Lee K, Whyment AD, Blanks AM, Spanswick D (2004) Orexigen-sensitive NPY/AgRP pacemaker neurons in the hypothalamic arcuate nucleus. Nat Neurosci 7:493-494.

Villarroel HS, Bompolaki M, Mackay J, Tapia AP, Michaelson SD, Leitermann RJ, Marr RA, Urban JH, Colmers WF (2018) NPY induces stress resilience via down-regulation of Ih in principal neurons of rat basolateral amygdala. J Neurosci :3528-3517.

Vollmer LL, Schmeltzer S, Schurdak J, Ahlbrand R, Rush J, Dolgas CM, Baccei ML, Sah R (2016) Neuropeptide Y impairs retrieval of extinguished fear and modulates excitability of neurons in the infralimbic prefrontal cortex. J Neurosci 36:1306-1315.

Wallace MN, Shackleton TM, Palmer AR (2012) Morphological and physiological characteristics of laminar cells in the central nucleus of the inferior colliculus. Front Neural Circuits 6:55.

West MJ, Slomianka L, Gundersen HJ (1991) Unbiased stereological estimation of the total number of neurons in the subdivisions of the rat hippocampus using the optical fractionator. Anat Rec 231:482-497.

Widdowson PS (1993) Quantitative receptor autoradiography demonstrates a differential distribution of neuropeptide- $\mathrm{Y}$ Y1 and Y2 receptor subtypes in human and rat brain. Brain Res 631:27-38.

Winer JA, Saint Marie RL, Larue DT, Oliver DL (1996) GABAergic feedforward projections from the inferior colliculus to the medial geniculate body. Proc Natl Acad Sci USA 93:8005-8010.

Wirth MJ, Patz S, Wahle P (2005) Transcellular induction of neuropeptide Y expression by NT4 and BDNF. Proc Natl Acad Sci USA 102:3064-3069.

Xu J, Kirigiti MA, Cowley MA, Grove KL, Smith MS (2009) Suppression of basal spontaneous gonadotropin-releasing hormone neuronal activity during lactation: role of inhibitory effects of neuropeptide Y. Endocrinology 150:333-340.

Zeller K, Rahner-Welsch S, Kuschinsky W (1997) Distribution of Glut1 glucose transporters in different brain structures compared to glucose utilization and capillary density of adult rat brains. J Cereb Blood Flow Metab 17:204-209.

Zeng H, Sanes JR (2017) Neuronal cell-type classification: challenges, opportunities and the path forward. Nat Rev Neurosci 18:530-546. 